\title{
Doubles traductions et omissions: une approche critique en vue d'une édition de la traduction latine du Liber de causis
}

\author{
Jules Janssens \\ De Wulf-Mansion Centre, Leuven
}

Il y a presque trente ans, Richard Taylor publiait une liste de soixante-quinze cas dont la grande majorité concernait la nécessité de revoir de façon majeure (ou, dans des cas plus rares, mineure) l'édition de la traduction latine du Livre du Bien pur par A. Pattin ${ }^{1}$, quelques cas ayant trait directement au rapport entre la traduction latine et le texte $\operatorname{arabe}^{2}$. Ces remarques étaient largement dérivées de sa thèse de doctorat ${ }^{3}$, qui signalait, pourtant, un plus grand nombre de cas impliquant une divergence entre la traduction latine et le texte arabe tel qu'il l'avait édité4. Plus que quiconque, Taylor était conscient des nombreux problèmes auxquels tout éventuel nouvel éditeur ou réviseur de la traduction latine est inévitablement confronté: le nombre extrêmement limité des manuscrits arabes (un seul connu à l'époque de l'édition Pattin, trois à l'époque de l' édition Taylor), et à l'inverse un nombre très élevé de manuscrits latins ${ }^{5}$. Ensuite, il y a la nature particulière du texte lui-même, qui constitue dans son ensemble une paraphrase d'une série de propositions des Éléments de théologie de Proclus, non sans certains remodelages doctrinaux et mise à contribution d'autres sources, en particulier la Pseudo-Théologie ${ }^{6}$. Un rapprochement avec le texte des Éléments de théologie peut donc être utile pour

1 Pattin 1966, p. 134-203.

2 Taylor 1989, p. 84-10o. Taylor (1989, p. 84) reconnaît que son projet est similaire à celui de Vansteenkiste 1967 , mais remarque à juste titre que ce dernier prenait comme référence pour l'arabe l'édition Bardenhewer (basée sur le seul manuscrit Leyde Or. 2o9) et ignorait donc les manuscrits Ankara, İsmail Saib I 1696 et Istanbul, Sülemanyie Kütüphanesi, Hacı Mahmud 5683. Notons toutefois que le manuscrit d'Istanbul est une copie directe de celui d'Ankara, voir Taylor 1982, p. 259-262 (nous remercions M. Taylor d' avoir attiré notre attention sur son article où il offre sinon la preuve, du moins des indications très fortes en faveur d' une telle dépendance).

3 Taylor 1981, p. 431-519.

4 Taylor 1981, p. 130-279.

5 Pour une liste provisoire, énumérant 237 manuscrits, voir Taylor 1983, p. 68-8o.

6 D’Ancona 1995, p. 121-153; voir aussi Taylor 2016, p. 228-23o. 
une compréhension correcte du texte, et apporter ainsi une contribution à l'évaluation adéquate de certaines différences entre le texte arabe et la traduction latine, bien que limitée aux cas où le rapprochement avec le texte grec de Proclus est incontestable.

L' existence d' une autre version arabe du Livre du Bien pur, que Thillet et Oudaimah ont appelé Liber de causis II, présente également un intérêt manifeste $^{7}$. Il faudrait aussi tenir compte de la réception dans la tradition arabe, fût-ce de façon partielle, du Liber de causis I dont témoignent: le vingtième chapitre du Livre de la Métaphysique de 'Abd al-Lāțif al-Bag̉dādīi ; la seconde partie de la Réfutation de celui qui parle de la disparition et l'annihilation de l'âme après la mort, le plus souvent attribué à Platon, mais parfois à al-Fārāb $\overline{1}^{9}$; plusieurs paragraphes des Chapitres sur des marques distinctives divines (Fușūl) d'al-'Āmirî' ${ }^{10}$; quelques fragments dans les Questions siciliennes d'Ibn Sab'īn ${ }^{11}$; et enfin des larges extraits du Ce qui est nécessaire pour celui qui connaît (Budd) de ce même auteur ${ }^{12}$. La traduction latine est parfois conforme à une leçon

$7 \quad$ Liber de causis II.

8 Badawi 1955, p. 248-256; Taylor 1981, p. 520-529. Une présentation brève de ce chapitre se trouve chez Martini-Bonadeo 2013, p. 242-254, où il est indiqué (p. 243) qu' al-Bag̉dādī reproduit, dans l' ordre, (des extraits de) tous les chapitres du Livre du Bien pur, à l' exception des chapitres 4, 10, 18 et 20. Taylor (Taylor 1981, p. 120) tient compte du texte tel qu' al-Bag̉dādī le présente - non de façon systématique, mais de temps à autre.

9 Türker 1965, p. 58, § 2-59, §3; Badawi 1974, p. 338-339; Taylor 1982, p. 530-544. Ce petit traité contient une citation (relativement littérale) du 'chapitre' 23 et de la première partie du chapitre 5 (et a été mis à contribution par Taylor pour l'édition de ces 'chapitres', voir Taylor 1982, p. 120).

10 Wakelnig 2006; Taylor 1981, p. 545-548. Notons que Wakelnig indique quelques parallèles en plus de ceux déjà signalés par Taylor (Taylor 1981, p. 546) et que les Fuṣūl citent toujours de façon très fragmentaire et non littérale les 'chapitres' du Livre du Bien pur (rapprochements avec tous les 'chapitres', à l' exception des chapitres 5, 10, 13-17, 20, 27, 28 et 31).

11 Akasoy 2006, p. 340-41o (arabe), 411-563 (traduction allemande); Spallino 2002, p. 314-223 (reproduction de l'édition arabe de Yaltkaya 1941), 53-222 (traduction italienne); Taylor 1981, p. 549-553, où une liste des citations du Livre du Bien pur est donnée (liste ignorée autant par Akasoy que par Spallino).

12 Ibn Sab'in 1978, passim; Lator 1944, p. 415-417 établit une liste des passages où le Budd cite un 'chapitre' (en entier ou partiellement), faisant référence au manuscrit Carullah 1273 d'Istanbul pour le Budd et à l'édition Bardenhewer 1882 pour le Livre du Bien pur, mais nous avons trouvé quelques passages additionnels (indiqués en gras dans la liste qui suit). Voici une nouvelle liste (toujours provisoire) des fragments communs, faisant référence aux éditions Taylor 1981 [T] (et Badawi 1955 [B]) pour le Kitāb 'al-īdāh fi hayr al-maḥ, Livre du Bien pur et à l'édition Katturah [K] pour le Budd: 1: T p. 138,11-13 (B p. 3,10-12) // K p. 77,23-24; 2:T p. 144-146 (B p. 4-5) // K p. 50,3-16 (incluant quelques additions et changements mineurs dans la formulation); 3: T p. 147,1-148,11 (B p. 5,10-14) // K p. 132,9-14; 4: T p. 151,1-152,8, 156,33-159,48 et 159,49-52 (B p. 6,8-11, 7,10-8,6 et 8,7-9) // K p. 49,15-21, 202,3-13 
non attestée dans les trois manuscrits utilisés par Taylor pour son édition, mais pourtant présente dans un (ou plusieurs) de ces ouvrages que nous venons de mentionner, comme nous le montrerons dans la suite. Quant à la réception de la traduction latine elle-même, elle fut considérable et selon l'état actuel des recherches, on dénombre une soixantaine de commentaires entre le douzième et le seizième siècle ${ }^{13}$. Ils ont clairement une importance capitale pour l'étude de la transmission de la traduction latine, car ils ne sont pas dépourvus de valeur pour fixer la leçon originale la plus vraisemblable. Enfin, une édition critique de la traduction latine ne peut pas ignorer l' existence des trois traductions latino-hébraïques ${ }^{14}$.

Mais avant d' offrir des exemples illustrant l'importance de ces multiples témoins indirects, nous voulons attirer l'attention sur quelques phénomènes typiques des traductions arabo-latines tolédanes de la fin du XII ${ }^{\mathrm{e}}$ siècle. Une partie importante fut réalisée par le cercle de Gérard de Crémone, dont la paternité de la traduction du Livre de la bonté pure ne semble plus être contestée à l'heure actuelle.

Doubles traductions

Le phénomène des doubles traductions a été bien attesté dans les volumes publiés de l'Avicenne latin: De anima, De prima philosophia et Liber primus naturalium, Tractatus I et II. Les traductions rapportées dans ces volumes datent toutes $\mathrm{du} \mathrm{XII}{ }^{\mathrm{e}}$ siècle et toutes ont été faites à Tolède, bien que dans le cercle de Gundissalinus. Simone Van Riet a eu le mérite d' avoir attiré l' attention sur lui. Elle en a conclu à l' existence des deux familles, dont l'une reflète la traduction originale, l' autre un effort de révision de celle-ci, soit par un copiste,

et 202,19-21; 6: T p. 166-170 (B p. 9-10) // K p. 215,11-216,3; 9: T p. 181,2-184,18 (B p. 12,19-13,7) // K p. 200,22-201,10; 12: T p. 192,2-193,8 (B p. 14,15-15,2) // K p. 51,24-52,4; 13: T p. 195,2-5 (B p. 15,7-9) // K p. 52,11-14; 14: T p. 200-202 (B p. 16) // K p. 38,15-20 (quelques modifications mineures dans la formulation); 20: T p. 229-231 (B p. 22)// K p. 133,22-134,6; 21: 20: T p. 232-234 (B p. 22-23) // K p. 133,12-20; 22 : T p. 235,2-236,7, 237,11-12 et 238,26-30 (B p. 23,710,12-13 et 23,15-24,1) // K p. 147,21-148,6; 23: T p. 239,2-24O-10 (B p. 24,3-7) // K p. 148,7-13; 25: T p. 248-251 (B p. 26) // K p. 134,7-20 (aussi p. 312,1-14); 26: T. p. 252-253 (B p. 27) // K p. 148,15-2O; 28:T p. 258-259 (B p. 28) // K p. 312,17-22; 31:T p. 273,2-274,12 (B p. 31,11-32,4) // K p. 133,4-10.

13 Calma 2016a, p. 20-21.

14 Concernant ces trois traductions et leur signification comme témoins de la traduction latine, voir Rothschild 1994 et Rothschild 2013. 
soit par le traducteur lui-même, en vue d' établir une traduction plus littérale ${ }^{15}$. Nous avons essayé de démontrer que ces doubles traductions furent sans doute présentes dans l'exemplaire original même du traducteur et qu' elles résultaient d' un effort pour 'latiniser' le premier essai de traduction (trop) littérale ${ }^{16}$. Quelle que soit l' alternative que l' on accepte parmi ces deux hypothèses, il est incontestable qu' il est possible, pour chacune de ces traductions, de distinguer l'existence des deux familles dans leur transmission. Pour chaque traduction on assiste, en outre, à l' existence de manuscrits contaminés, où des variantes spécifiques à chacune des deus familes sont attestées. L'étude systématique des doubles traductions est donc utile afin de découvrir si l' on est bien confronté à ce qui peut être qualifié de 'familles'. Comme il est incontestable que les nombreux manuscrits de la traduction latine du Livre du Bien pur portent beaucoup de doubles traductions, il est clair qu' il faut y prêter une attention sérieuse, car celles-ci peuvent contribuer à mieux saisir sous quelles différentes formes la traduction latine a été transmise, voire, ne fût-ce dans une mesure limitée, à établir le stemma codicum. Sans être exhaustif, nous proposerons dans ce qui suit quelques cas exemplaires et/ou significatifs. Nous nous limiterons en général, mais non toujours, à l'édition Pattin et à son apparat de variantes.

Nous commencerons avec un cas plutôt classique, à savoir ergo / igitur pour la conjonction arabe $f a$ - L'édition Pattin, en conformité avec six des dix manuscrits utilisés pour l' édition, porte en I, 9,23, ergo, mais l' expression équivalente igitur est attestée dans les quatre autres; la même variante est attestée après, à savoir en I, 18, 63 (trois manuscrits dont seulement deux la portaient aussi à la première occurrence), en $\operatorname{XIV}(\mathrm{XV}), 125,53$ (un seul manuscrit où elle figurait aussi dans le premier cas, mais pas dans le deuxième), $\mathrm{XXI}(\mathrm{XXII}), 168,77$ (un manuscrit, qui porte cette variante seulement ici), $\mathrm{XxIX}(\mathrm{xxx}), 204,84$ (cinq manuscrits, dont un l'attestait aussi dans le premier et le deuxième cas; un dans le premier et le troisième cas et enfin, un dans le seul premier cas, alors que les deux autres portaient toujours auparavant ergo), $\mathrm{xxIX}(\mathrm{xxx}), 206,7$ (un seul manuscrit, qui le portait aussi dans le premier, le troisième et le cinquième cas) et $\operatorname{XXXI}(\mathrm{XXXII}), 219,12$ (deux manuscrits, dont l'un en témoignait dans le premier, le troisième et le cinquième cas, et l' autre dans le deuxième et le cinquième cas). Inversement, on trouve igitur dans le texte et ergo dans l'apparat des variantes en I, 12, 39 (ergo: quatre manuscrits, dont deux figuraient aussi comme témoins de la variante igitur pour ergo) et en III, 36, 32 (ergo: un manuscrit, qui fait partie des quatre témoins du premier cas). Sans en spécifier

15 Voir Van Riet 1963; Avicenna latinus 1977, p. $128^{*}-130^{*}$ et Avicenna latinus 1992, p. $54^{*}-62$.

16 Voir Janssens 2002. 
le détail, mentionnons encore d' autres variantes pour ergo, telles que autem, enim, unde et vero. Mais le cas de ergo / igitur suffit à mettre en lumière qu' il existe une assez grande fluidité entre les deux, ce qui se laisse aisément expliquer si ce choix fut effectivement présent dans l'autographe du traducteur.

Un cas très significatif, car très fréquent, est la traduction de l' adjectif arabe 'aqli par un nombre assez important de termes différents: intellectibilis / intelligibilis / intellectualis / intelligentialis / intelligens / intelligentiae / intellectus ${ }^{17}$. L'édition porte dans la vaste majorité des cas (une quarantaine) intellectibilis. Mais il est frappant que l' apparat des variantes mentionne partout l' existence, dans au moins un des dix manuscrits témoins, de la variante intelligibilis et que celle-ci soit tout simplement la leçon de la majorité des manuscrits ${ }^{18}$. À plusieurs reprises, on trouve un troisième terme: intellectualis (dix cas), intelligens, intelligentiae, intelligibiliter et intellectus (chaque fois dans une seule instance). La présence de deux autres options outre celle d' intelligibilis est attestée une fois: intellectualis et intelligentialis. Par contre, les deux mots intellectibilis et intelligibilis figurent dans l'apparat des variantes, bien qu' une seule fois (en III, 36,34), alors que le textus porte intelligentiae. Ce dernier cas a de quoi surprendre, mais il est relativement bien attesté et par ailleurs confirmé par le commentaire de Gilles de Rome ${ }^{19}$. On peut se demander si, à l' origine, on ne lisait pas dans la traduction eius intelligentiae / intellectibilis ou intelligibilis ${ }^{20}$, suivant à une hésitation selon laquelle il aurait fallu lire en arabe (quwwa) 'aqlihi ou 'aqliyya, car immédiatement après se trouve un cas similaire: l' arabe (quwwa) dātiyya est traduit par (virtutem) eius essentiae (ce qui suggère la lecture dâatihi) / (virtutem) essentialem (bien qu'attesté dans un manuscrit seulement $)^{21}$. Observons toutefois que dans le cas d' intelligentiae, contrairement à

17 Pour ne pas compliquer inutilement les choses, nous nous limitons à utiliser le nominatif singulier et ne tenons donc pas compte du cas de déclinaison. Nous ne faisons pas non plus attention à des coquilles évidentes, comme intellitibilem (III, 34, 20).

18 La raison de la préférence accordée par Pattin à la leçon intellectibilis n' est pas claire. Il est regrettable qu'il n' ait pas explicité la raison de son choix.

19 Gilles de Rome, Super Librum de causis, f. 12v-13rAA.

20 De nouveau, Pattin ne justifie nulle part son choix (comparez supra, note 18). Pourtant, ni les témoins manuscrits ni les commentaires choisis par lui pour son édition ne permettent de privilégier la leçon intelligentiae.

21 Pace Taylor 1989, p. 89, cas (14), nous ne croyons pas que le modèle arabe du traducteur latin portait seulement une leçon différente en ce qui concerne la troisième qualification, à savoir da ātihi au lieu de dātiyya. Observons que l'apparat de Pattin affirme l' existence dans ce dernier cas d'essentialis dans le manuscrit Vat. Lat. 20984 - unique, il est vrai indiquant ainsi que le traducteur latin, ou, à la limite, quelqu' un qui aurait révisé la traduction originale, lisait sans doute déatiyya, en conformité à la tradition manuscrite arabe (comme ce même manuscrit témoigne auparavant de la leçon intelligentiae, il semble 
celui d'essentiae, la variante intelligibilis est présente dans un bon nombre de témoins (quatre manuscrits, l'édition latine de Bardenhewer et le commentaire de Bacon).

Quant à la variante intellectibilis, elle est attestée dans le manuscrit Vat. Ottob. Lat. 1415, dont Vansteenkiste a démontré le caractère relativement précieux comme témoin du texte arabe ${ }^{22}$. Or, depuis que Taylor a élaboré une édition non seulement sur la base du manuscrit de Leyde, mais aussi de ceux d'Ankara et d' Istanbul, il est apparu évident que le manuscrit d' Aoste, Seminario maggiore 71 (olim $\mathrm{Ai}^{\circ} \mathrm{D} 20$ ) est lui aussi un témoin de grande valeur ${ }^{23}$. Il est dès lors incontestablement frappant de voir que lui aussi porte la leçon (virtutem) intellectibilem, exactement comme le manuscrit Vat. Ottob. Lat. 1415. Dans le cas présent, on peut par conséquent s'imaginer que l'archétype portait: et virtutem intelligentiae / intelligibilem intellectibilem, où d'une part on trouve un essai de traduction alternative suite à une hésitation entre une lecture 'aqliyya et 'aqlihi de l'arabe (de graphie assez similaire, surtout en absence de point diacritiques), et d' autre part l' alternative habituelle intellectibilis / intelligibilis. En outre, la leçon intellectibilis est (presque) partout ailleurs présente dans les deux manuscrits. Tout indique donc que ce terme, typique du néoplatonisme latin ${ }^{24}$, soit se trouvait dans l' archétype du traducteur, sans doute accompagné de l'alternative intelligibilis, soit que l'un de ces deux termes a été introduit comme alternative pour l' autre relativement tôt dans la transmission de la traduction. Parmi toutes les autres options, la variante intellectualis est encore relativement bien attestée et il est intéressant de constater qu' intellectualis se trouve dans le De anima de l'Avicenne latin (qui, bien sûr, n'appartient pas à Gérard de Crémone, mais à Gundissalinus et à son cercle) parmi les termes qui traduisent ${ }^{\prime} a q l_{\imath}{ }^{25}$. Le terme intelligentialis est certainement aussi une tra-

exclu que cette leçon d' essentialis soit due à une initiative de son copiste). En plus, comme nous l'avons dit, on peut aisément s' imaginer un doute chez le traducteur concernant la façon dont on doit lire l' arabe (le même problème se posant pour dātiyya/dātihi que pour 'aqliyya / 'aqlihi). Enfin, sa reconstruction et secundum virtutem intelligibilem implique une préférence pour intelligibilem à l' intérieur de l' alternative intellectibilis / intelligibilis. Mais, sauf erreur de notre part, rien ne justifie une telle préférence.

22 Vansteenkiste 1967, p. 62-66.

23 Concernant la valeur précieuse de ce manuscrit, voir Taylor 1989, p. 84 et 101-102. Quelques caractéristiques particulières de ce manuscrit avaient été déjà mises en lumière par Pattin 1966, p. 104-105.

24 Vansteenkiste 1967, p. 76.

25 Avicenna latinus 1968, p. 8,99 et 28,84 (mais ce terme n' est pas utilisé dans Avicenna latinus 1972). Observons encore que la variante intellectibilis ne figure dans l'ensemble de cette traduction qu' une seule fois, à savoir Avicenna latinus 1968, p. 153,11 (en alternative avec intelligibilis). 
duction valable, mais somme toute, un mot assez rare; il n' apparaît qu'une seule fois dans un seul manuscrit ${ }^{26}$. Quant à l'utilisation en VII(VIII), 78,45 de l'adverbe intelligibiliter, attestée dans un seul manuscrit, elle s' explique par le contexte, mais il faudrait alors s' attendre à la suppression du substantif apprehensione, qui précède intellectibili dans l' édition ${ }^{27}$. Par contre, les termes intelligens et intelligentiae posent problème. Le dernier correspond à une leçon arabe (al-) 'aql, mais l'utilisation d'intelligentiae pourrait résulter de la présence du même mot immédiatement à sa suite, alors que le premier présuppose une lecture 'āqil de l' arabe. Il est intéressant de noter que ces deux variantes ne sont attestées qu'une seule fois, à savoir en III, 34,19-20, à l'intérieur d'une seule phrase et semblent donc intimement liées ${ }^{28}$. Enfin, tout indique que la mention d'intellectus (en XVIII(XIX), 149,69), attestée dans un seul manuscrit (le ms. Bruges, Bibliothèque de la ville 463), résulte d'une erreur de copiste, car l'expression anima intellectus est dépourvue de sens. Selon nous, sur la base de toutes les observations qui précèdent, une nouvelle édition critique devra sans doute maintenir l' alternative intellectibilis / intelligibilis dans le textus. Mais, si sur la base du stemma codicum, il est incontestable qu' un des deux termes fut présent d'abord sans alternative et que l'autre a été introduit à un moment postérieur de la transmission de la traduction, alors on pourrait opter pour ne maintenir que le terme concerné dans le textus en vue d'éditer la traduction originale, ou, du moins, ce qui s' en rapproche le plus.

Un autre cas intéressant est la traduction des comparatifs arfa' et 'alā. En $\mathrm{v}(\mathrm{vI}), 63,61-63$, l' édition, à la ligne 61, porte dans le textus altior comme traduction d' $\operatorname{arfa}{ }^{c}$ et mentionne comme variante sublimior dans l' apparat, en faisant référence à un seul manuscrit, le ms. Tolède, Cabildo 97-1 (sur l'ensemble de dix

26 Le terme intelligentialis ( $\mathrm{x}, 102,62)$ se trouve dans le manuscrit Toledo, Cabildo 97-1. Il est attesté dans les traductions des œuvres de Proclus par Moerbeke, voir, par exemple, Procli opuscula, p. 120, 6, 10, mais il faudra examiner s'il ne fut déjà pas utilisé par Gérard de Crémone et son cercle. En effet, il semble possible qu'il s' agisse d'un choix du copiste inspiré par une volonté de souligner davantage le caractère proclien de l' ouvrage.

27 Le texte arabe porte: yudriku (...) idrākan 'aqliyyan, dont apprehendit (...) apprehensione intellectibili (ou: intelligibili) constitue une traduction très littérale. De façon plus latinisée, on attendrait plutôt une traduction apprehendit (...) intelligibiliter. Dans le contexte, on peut donc considérer apprehensione intellectibili (intelligibili) et intelligibiliter comme un essai de double traduction.

28 Elles sont toutes deux présentes dans les manuscrits Vat. Lat. 2089 et Bruges, Bibliothèque de la Ville 478, mais dans ce dernier intelligens figure en marge, comme correction pour intelligibilis et est écrit d' autre main que celle du copiste original. En III, 3420, la variante intelligentiae se trouve confirmée en outre par le manuscrit Bruxelles, Bibliothèque royale II, 2314. 
manuscrits qui constituent la base de l'édition). ${ }^{29}$ Toutefois, deux lignes plus loin, le textus offre la traduction sublimior, présente dans tous les dix manuscrits, dont, selon l' apparat, trois (Vat. lat. 2984, Vat. Urb. lat. 206 and Paris, BnF Lat. 6318) ajoutent subtilior comme traduction alternative. En VI (VII), 67,76 , on trouve la traduction altior et superior (sublimior dans deux manuscrits) pour l' expression arabe arfa'wa-'alā et, en XXI (XXII), 171,89 superior et altior (mais dans un manuscrit altior et superior) comme traduction d'alā wa-

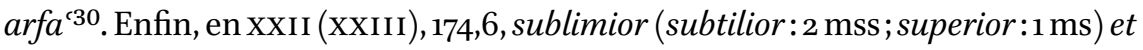
altior peut correspondre soit à la leçon $\operatorname{arfa}^{c} w a$ - $^{\prime} a l \bar{a}$, présente dans les manuscrits d'Istanbul et d'Ankara, soit à celle inversée, 'alā wa-arfa', attestée dans le manuscrit de Leyde ${ }^{31}$. À l' exception du premier cas, altior apparaît toujours sans alternative, alors que sublimior est accompagné deux fois de l'alternative subtilior, et une fois, en plus, de la variante superior, qui, à son tour, est clairement distingué d'altior en XXI(XXII), 171,89 (en outre, en V(VI), 57, 22, superior, sans variante aucune, traduit 'alā). On aurait donc tendance à croire que sublimior (subtilior / superior) traduit 'alà et qu'altior rend arfac. Par conséquent, le témoignage unique - parmi les dix manuscrits consultés par Pattin - du manuscrit Tolède, Cabildo 97-1, de la leçon sublimior comme alternative pour altior en $\mathrm{v}(\mathrm{VI}), 63,61$, pourrait paraitre à première vue suspect. Cependant, on constate immédiatement à sa suite que sublimior / subtilior traduit effectivement $\operatorname{arfa}$. Le traducteur latin aurait-il lu à cet endroit dans son modèle arabe 'alā ? Dans l'état actuel des choses, nous devons laisser cette question ouverte $^{32}$, mais il faudra essayer à l' occasion d' une nouvelle édition critique de résoudre ce problème délicat, surtout en vue de fixer les lexiques arabe-latin et latin-arabe.

29 Bien qu' il énumère 92 manuscrits, Pattin s' est limité pour son édition à collationner entièrement dix manuscrits. Bien qu'il précise lui-même que son édition ne prétend pas être une édition critique définitive, il est regrettable qu'il n' ait pas expliqué les raisons de ce choix. En outre, dans quelques cas rares il prend recours à d'autres manuscrits. Là encore, il n'explique pas clairement ce qui motive son choix. Notons simplement qu'il s' est limité à collationner, pour le passage sous discussion, les dix manuscrits témoins majeurs.

30 Certes, on lit altior dans le textus de l'édition et superior dans l'apparat des variantes (un manuscrit), mais il ne s' agit là pas d'une vraie variante, car il y a eu inversion des deux termes. En fait, le copiste du manuscrit Vat. Lat. 2089, a clairement inversé les deux termes altior et superior, qui se trouvent tous deux très proches sur la même ligne.

31 Pour l' existence de cette lecture inversée dans la tradition arabe, voir Taylor 1981, p. 237, note 12, où il met en parallèle la traduction latine avec la leçon du manuscrit de Leyde.

32 Sauf erreur de notre part, aucun témoin arabe, direct ou indirect, ne contient une indication à cet endroit de la leçon 'ală. 
Un cas qui mérite une attention très particulière est celui de l'expression non destruuntur [nec permutantur] en $\mathrm{x}(\mathrm{XI}), 101,55-56$. Dans le texte arabe on lit seulement lā tastahĭlu, ce qui explique que Pattin ait opté pour la suppression de nec permutantur. Taylor a estimé que nec permutantur traduit sans doute lā tatagayyaru, expression attestée dans le manuscrit de Leyde, mais seulement à la fin de l'affirmation qui suit dans le texte ${ }^{33}$. Il incline donc à y reconnaître un cas où la traduction latine témoigne d'un déplacement d'un segment de texte par rapport à l'arabe. $S$ 'il s'abstient d'offrir une explication pour ce déplacement, on pourrait songer à une mention de nec permutantur dans la marge de la copie du traducteur, qui, à cause d'un oubli, aurait marqué par un signe que l' expression devait être ajoutée; après, lors de la transmission de la traduction, l'endroit du signe aurait été mal interprété par un copiste. Cette explication est en principe possible, mais loin d'être évidente. Pattin et Taylor semblent avoir perdu de vue que le verbe permutantur rend parfaitement le sens du verbe arabe tastahïlu, même plus que ne le fait le verbe destruuntur $^{34}$. En outre, un seul des dix manuscrits utilisés pour l'édition porte nec permutantur; quatre, par contre, lisent vel permutantur et cinq omettent l'expression ${ }^{35}$. Comme Vansteenkiste l'avait remarqué il y a déjà un demisiècle, permutantur résulte sans doute d'un essai de double traduction par le traducteur lui-même et l'utilisation de la conjonction vel en constitue un indice sérieux ${ }^{36}$. Compte tenu de tous ces éléments, nous sommes plutôt convaincus

33 Taylor 1981, p. 466, note 88. En Taylor 1989, aucune remarque est formulée à propos du passage.

34 La traduction du verbe istahăla par permutari est solidement établi dans le De anima de l'Avicenne latin, voir Avicenna latinus 1972, p. 311 et Avicenna latinus 1968, p. 229, alors que dans le De philosophia prima, la traduction permutari est absente bien que, il est vrai à une seule occasion, le substantif istihăla y est rendu par permutatio - par contre, on y trouve à deux reprises la traduction alternative destrui (absente du De anima), voir Avicenna latinus 1983, p. 34 .

35 Un rapide survol d'une vingtaine de manuscrits, outre ceux consultés par Pattin, nous a montré que le cas de l'omission est le mieux attesté dans la tradition manuscrite (y compris dans le manuscrit Aoste, Seminario maggiore 71). Elle est confirmée par les commentaires de Thomas d'Aquin et de Gilles de Rome et, en plus, par deux des trois traductions latin-hébraïques (mais la troisième de celles-ci confirme la leçon nec permutantur), voir Rothschild 1994, p. 445. Toutefois, la traduction vel permutantur se trouve, outre dans les quatre manuscrits désignés par Pattin, dans quelques autres, tels par exemple, Troyes, вм 1374; Rouen, Bм 920; Chicago, Vault Case 23.

36 Vansteenkiste 1967, p. 63, où il note: «l'arabo ha soltanto un verbo (che significa permutantur, ma che viene anche tradotto con corrumpere e destruere); l' espressione sembra essere dunque un doppione, forse dello stesso traduttore, e potrebbe essere stato con vel, conservato da O [= Vat. Ottob. Lat. 1415]». Pour le rôle des conjonctions dans la formulation de doubles traductions, voir Janssens 2002, p. 118-120. 
que la leçon non destruunturvel permutantur était présente dans l'exemplaire du traducteur, qui hésitait entre ces deux verbes latins pour savoir lequel rendait le mieux l' arabe - le second ayant l' avantage d' être plus littéral, le premier d'être plus adapté au sens général du chapitre. On comprend aisément qu'un copiste ait cru que ce vel soit inadéquat dans la mesure où il semble suggérer que les choses éternelles soit ne sont pas détruites, soit subissent un changement, et, afin d'arriver à une affirmation plus cohérente, l'a remplacé par neque. Un autre copiste a sans doute estimé que l' alternative était trop littérale et l'a par conséquent supprimée ${ }^{37}$. Il est clair que c'est cette dernière version qui a été la plus répandue. Mais, somme toute, il est hautement probable que l'expression non destruunturvel permutantur corresponde le mieux à la traduction originale. Selon le poids que l' on accorde tantôt à approcher au maximum la traduction d' origine, tantôt à valoriser la réception de cette traduction, on éditera donc soit la formule telle que nous l' avons exprimée, soit on se limitera à non destruuntur. En aucun cas, la formule non destruuntur neque permutantur, que Pattin a choisie (tout en mettant entre crochets neque permutantur) ne mérite de figurer dans le textus ${ }^{38}$.

Enfin, nous ne voulons pas passer sous silence les multiples occasions où l'on trouve, dans un ou plusieurs manuscrits, causare / causatum / causans au lieu de creare / creatum / creans, ou alors, bien que ce soit plus exceptionnel, l'inverse. On pourrait spontanément songer à un essai de traduction double (ou alternative). Généralement parlant, la variante qui ne correspond pas vraiment au terme original arabe (causare, etc. fautivement pour $b d$ ' et creare, etc. fautivement pour ' $l l$ ) n' est attestée que dans un nombre plutôt infime de manuscrits. Tout indique qu' il s' agissait donc d'une confusion faisant suite à une lecture fautive qui a eu lieu au cours de la transmission de la traduction (la confusion entre les deux termes latins étant somme toute courante en raison des abréviations $)^{39}$. Cependant, une étude approfondie à ce sujet est souhaitable et reste donc à faire.

\footnotetext{
37 Nous avons observé le même phénomène dans l' Avicenne latin, voir Janssens 2002, p. 118.

38 Si la traduction latine offre effectivement un essai de double traduction, elle correspond pleinement au texte arabe, tel qu' il est attesté dans les trois manuscrits connus.

39 Il est d'un intérêt particulier de remarquer que dans la Philosophia prima de l' Avicenne latin, ne figure qu'une seule occurrence (Avicenna latinus 1980, p. 481, 51) de causatis là où creatis était normalement attendu, ce qui conduit plutôt vers une lecture fautive que vers une réelle double traduction ou traduction alternative. De façon plutôt surprenante, S. Van Riet n' offre aucune note.
} 


\section{$2 \quad$ Traductions alternatives (de type exclusif)}

La traduction de la conjonction $w a$ - dans une et même occurrence par et et sed peut être considérée comme l' expression d' un essai de traduction alternative. Ce cas se présente en I, 11,31 et $33^{40}$.

L'expression [apponitur vel] parificatur (II, 25,91), retenue par Pattin, est sans doute beaucoup plus significative, même si aucun des dix manuscrits témoins ne la porte ${ }^{41}$. Toutefois, la formule apponitur vel parificatur est attestée dans quelques manuscrits, tels que: Assise, вс 663; Berlin Staatsbibliothek, M 1494, Lat. Qu. 449; Munich, BsB Clm 527 et Rennes, Bм 149. Mais la tradition manuscrite révèle l' existence d'une variété nettement plus grande:

- opponiturvel parificatur:

Bruges, Bibliothèque de la Ville 463; Tolède, Bibl. del Cabildo 97-1; Paris, BnF, lat. 16o82 ; Florence, Bibl. Laur., Ashburnham 1674; Bologne, Bibl. Univ. 2344; Rouen, BM 920; Troyes, BM 1374; Bordeaux BM 421; Vienne, ÖNB 2491; Genève, BG E, Lat. 76 ; Sankt Gallen, Stiftsbibliothek, Cod. Sang. 837; Cava de' Tirreni (Salerne), B. ss. Trinità 31; Madrid, BN 489. Le ms. Aoste, Sem. Magg. 71: opponitur dans le texte ${ }^{42}$, mais ajoute de vel parificatur dans la marge.

- opponitur et parificatur:

Angers, BM 450

- opponitur / parificatur:

Bruges B C 102/125: opponitur dans le texte, parificatur au-dessus de la ligne; Cologny, Bodmer СB 10: opponitur dans le texte, parificatur (dans une autre encre) au-dessus de la ligne;

- opponitur:

Bruxelles, Bibliothèque Royale 2314; Assisi вC 298; Césène, Bibl. Malatestiana, Ms. Plut. XXII, Dext. 6; Paris, BnF, lat. 6318.

- opponitur exponctué, parificatur dans la marge:

Tours, вм 680.

- parificatur:

Vat. Lat. 2089; Oxford, Bodl., Selden sup. 24; Césène, Bibl. Malatestiana, Ms. Plut. XXIII, Dext. 6 ; Erfurt, Ca-2-oo363; Évreux, BM 79; Berlin M 1491, Lat. Qu. 48.

40 Taylor 1981, p. 438, notes 7 et 8 , observe à juste titre que la conjonction $w a-$ «may be translated here as sed or $e t »$.

41 Pattin 1966, p. 139, note 91, affirme que le verbe apponitur est attesté dans le manuscrit Vat. Urb. Lat. 206, ainsi que dans le commentaire de Gilles de Rome. Cette dernière affirmation est erronée, car Gilles lit clairement le seul verbe parificatur (Gilles de Rome, Super Librum de causis, f. 8vX).

42 Taylor 1981, p. 443, lit à tort apponitur. 


\section{- apponitur:}

Vat. Lat. 206; Vat., Ottob. Lat. 1415; Chicago, Vault Case Ms. 23. Notons encore que le manuscrit Erfurt, $\mathrm{Ca}-4-00018$ porte ponitur (pour opponitur?) et que le manuscrit Admont, cod. 405 est abimé à cet endroit, mais il semble que le copiste ait effacé un mot avant d'écrire opponitur au-dessus de la ligne. Ces manuscrits sont donc plutôt indicateurs d' une leçon qui se limite au seul verbe opponitur. Enfin, dans le manuscrit Leipzig, Universitätsbibliothek 1339 opponitur a été exponctué et remplacé par coextenditur dans la marge par une autre main et dans une autre encre ${ }^{43}$.

Nous inclinons à croire que le traducteur a hésité comment lire le verbe arabe: yuḥād $\bar{\imath}$ (opponitur) ou yuğārī (parificatur) ${ }^{44}$. En effet, en l'absence de points diacritiques, la graphie des deux verbes arabes est très similaire. La tradition manuscrite indique de façon assez nette que les deux verbes opponitur et parificatur étaient tous deux présents dans l'archétype, pourvus ou non de la conjonction vel. Dans la suite, à un moment qu'il est difficile voire impossible à déterminer, on voit la suppression d'un des deux verbes, conduisant à l'existence de deux nouvelles familles, dont l'une n' atteste que le seul verbe opponitur, l' autre que le seul verbe parificatur. Le manuscrit de Tours, Bм 680 est incontestablement éclairant pour ce développement, car son copiste a clairement les deux verbes sous les yeux, mais il exponctue opponitur et le rem-

43 Sans doute, sur la base du contexte (quoniam extenditur cum ea), un lecteur attentif du manuscrit aura saisi que la leçon opponitur fait problème et opté pour une correction en coextenditur, qui donne lieu, toutefois, à une affirmation assez redondante.

44 Les trois manuscrits arabes connus portent la leçon yuğārī sans points diacritiques, sauf que le manuscrit de Leyde vocalise le $y$ du début et suggère ainsi une lecture tuğārī (voir Taylor 1981, p. 146, l. 3 [Bāb, 22, l. 14]). Badawi, dans son édition (voir Badawi 1955, p. 5,

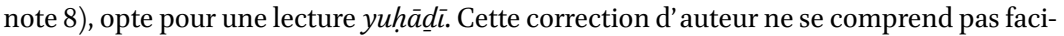
lement, car le contexte invite plutôt à lire yuğârī. Aurait-elle été faite sur la base de la traduction latine? D' autre part, nous doutons l' affirmation de Vansteenkiste (voir Vansteenkiste 1967, p. 62) selon laquelle le verbe ğărī peut avoir le sens d'opponere. Rothschild (Rothschild 1994, p. 415, note 6) affirme que le verbe opponere a ici le sens secondaire de «mettre en regard» et que ce sens est attesté en latin classique, mais nous n' avons trouvé que celui de «placer devant». En outre, d'après lui, les deux verbes opponere et parificari paraissent mieux correspondre à $h \bar{a} \underline{d} \bar{l} \bar{\imath}$, «être vis-à-vis de», ce qui nous paraît discutable. Signalons encore que dans cette même note, Rothschild indique que les trois traductions latino-hébraïques avaient dans leur modèle soit le seul verbe opponitur, soit le seul verbe parificatur: elles portent en effet trois formes différentes (šāwwōt, mešsuwwōt, yišetawwōt) de la racine $\check{s} w h$ qui signifie principalement l' «égalité» et de manière moins aisée, mais possible, la «mise sur un même pied », la « comparaison» (v. Klatzkin 1933 (en hébreu), t. IV, p. 78-8o); tant à cause du sens de la racine hébraïque que de celui des termes latins, le rapport avec parificatur paraît malgré tout le plus immédiat et le plus indiscutable. (Nous remercions très vivement $M$. Rothschild pour cette précision.) 
place - en marge, il est vrai - par l' autre verbe, à savoir parificatur. Quant à la leçon apponitur, elle résulte sans doute de la démarche d' un copiste intelligent qui a saisi que la leçon opponitur pose problème d'un point de vue doctrinal. Afin d'y 'remédier', celui-ci a choisi le verbe apponitur qui se rapproche davantage de parificatur, sans en être pour autant un parfait synonyme, croyant vraisembablement qu'opponitur était le fruit d'une lecture erronée d'apponitur par un copiste.

\section{$3 \quad$ Omissions}

Une partie importante des omissions suggérées dans l'édition Pattin sont basées sur la version du texte original tel que celui-ci a été préservé dans le manuscrit de Leyde. Souvent, pourtant, le latin se révèle ici conforme au texte arabe tel qu'il est attesté dans les manuscrits d'Ankara et d'Istanbul. Il en va ainsi pour I, 5,16; VI(VII), 69,86; $\mathrm{x}(\mathrm{XI}), 102,59$; XIII(XIV), 123,43 et 123,49; XVI(XVII), 139,20; XVII(XVIII), 148,62; XVIII(XIX), 154,95; XIX(XX), 158,19 et 159,31, 34; $\mathrm{xx}(\mathrm{XXI}), 164,59$ et 164,6o.

Des cas plus délicats constituent XXII(XXIII), 175,9 et XXIX(XXX), 204,78. Pattin y supplée chaque fois la négation non, ce qui s' impose en effet d' un point de vue doctrinal. Dans le premier cas, la négation lā est présente dans le manuscrit de Leyde (et confirmé par 'Abd al-Lāțif al-Bag̉dādī), mais pas dans ceux d'Ankara et d' Istanbul, alors que dans le second elle est absente de tous les trois témoins arabes. On peut donc se demander avec Taylor s' il est justifié de suppléer dans un cas comme dans l' autre la négation dans la traduction latine contre la tradition manuscrite arabe (surtout dans le second cas, où elle est unanime), et, ajouterions-nous, contre le témoignage du commentaire littéral de Gilles de Rome ${ }^{45}$.

Dans deux cas, il est possible qu' il y ait eu une omission volontaire de la part du traducteur car les affirmations sont assez redondantes:

45 Gilles de Rome, Super Librum de causis, f. 8orT et 1oovE. Avant d'apporter un jugement définitif, il faudra examiner en détail l'ensemble de la tradition manuscrite de la traduction latine. Observons encore que l'absence de la négation en XXII(XXIII), 175, 9, est confirmée par les traductions hébraïques de Juda, d'Ḥabilio (à partir de la traduction latine) et de Zerahyah (basée directement sur le texte arabe) (voir Rothschild 1994, p. 468, note 2) et celle en $\operatorname{xxIx}(\mathrm{Xxx}), 204,78$ par celles de Juda et de Zerahyah, mais pas de Habilio (Rothschild 1994, p. 479, note 3). Que ce dernier ait lu non dans son modèle, comme Rothschild le suppose, n' est pas absolument certain, car il se pourrait qu'il ait senti le besoin d'introduire la négation afin de sauvegarder la cohérence doctrinale. 
- en $\mathrm{X}(\mathrm{XI}), 102,61$, la spécification «qui tombent sous la génération et la corruption (al-wāqi'at tahtu l-kawn wa-l-fasād)», car elle exprime exactement la même idée que l'adjectif destructibiles, qui précède;

- en XXXII(XXXIII), 218,98, l' addition « et qu'il [à savoir un deuxième Un supposé en concurrence avec l'Un véritable] soit un comme lui (wa-kāna wāhidan mițlihi)», car il a déjà été affirmé que cet Un supposé ressemble à tous les égards à l'Un véritable.

Cependant, plutôt que de signaler une omission dans le textus, il nous semble préférable de formuler pour ces cas une note indiquant simplement que le texte arabe ajoute une affirmation redondante - ce qu' il est possible de faire dans un apparat qui discute le rapport précis entre le texte arabe et la traduction latine.

Quant à l'omission d'etiam (ar. aydan) en VI(VII), 70,9o et XV(XVI), 131,85, elle pourrait avoir sa source dans la transmission de la traduction latine. En effet, à cause de l' abréviation médiévale, un copiste a pu croire à un iteravit de la conjonction et qui précède. Mais elle pourrait aussi résulter d'un choix délibéré du traducteur ou encore de l'absence du terme aydan dans le modèle du traducteur. Si la première hypothèse s' avérait correcte et que l'on trouvait etiam attesté dans au moins un manuscrit, il serait préférable de l'introduire dans le textus et d'indiquer son omission dans un (grand) nombre de manuscrits. Mais il ne s' agit plus alors d'une omission. Quant aux deux autres hypothèses, elles reconduisent à l'idée d'une note dans un apparat séparé.

L'omission, qui est clairement présente en $\mathrm{IX}(\mathrm{x}), 96,33$, et qui est confirmée par les traductions latino-hébraïques ${ }^{46}$, résulte probablement d'un saut du même au même, lié au texte arabe dans le modèle du traducteur: inbijāsan kulliyyan mutawahhidan... inbijäsan kulliyyan mutawahhidan ${ }^{47}$. Si cela s' avère correct, le traducteur latin a lu: 'aruda min d̄âlika an takūna l-ṣuwar allatī tanbajasa min al-uqūl al-awwal (...) inbijāsan kulliyyan mutawaḥhidan. Il a pu alors considérer la séquence allatī... al-awwal comme une phrase relative qui précise de quel genre de formes (șuwar) il s' agit et a pu par conséquent, lier inbijāsan directement avec le verbe takūn - verbe qu'il traduit, conformément au contexte, par procedant plutôt que par la traduction habituelle sint. Il est néanmoins intéressant de voir que Gilles de Rome insiste dans son commentaire ${ }^{48}$ sur ce qu' implique de différent cette procession universelle des intellects premiers au niveau des intellects seconds: supple inde accidit quod ex intelligentiis secundis procedunt formae processione particulari multiplicata. Il couvre ainsi

\footnotetext{
$46 \quad$ Rothschild 1994, p. 442-443, note 1.

47 Nous remercions les participants du colloque d' avoir attiré notre attention sur l' extension précise de l'omission.

48 Gilles de Rome, Super Librum de causis, f. $38 \mathrm{rN}$.
} 
presque littéralement une large partie de l' omission où il est explicitement dit, dans le texte arabe, que les formes "procèdent des intellects seconds selon une procession particulière et multiple» (mss Ankara et Istanbul; ms Leyde: distincte $)^{49}$.

Un cas particulier concerne la proposition XVIII(XIX), 150,75. Comme ce cas fait partie d' une affirmation assez corrompue dans son ensemble, nous le discuterons plus tard, lorsque nous nous pencherons avec une attention particulière sur la complexité de la transmission de la traduction latine..$^{50}$

Enfin, le passage $\operatorname{XIX}(x x), 159,29-31$, pourrait laisser croire à une seconde omission à la même ligne 31 , non signalée par Pattin et qui serait à placer après la conjonction et. Mais la traduction latine soulève ici de larges difficultés. On peut y lire (selon l'édition Pattin): scilicet quando agens et factum sunt per instrumentum et [or cette conjonction est absente dans six des dix manuscrits témoins et devrait, selon nous, être supprimée] non facit per esse suum et sunt composita $^{51}$. Le texte arabe affirme: $a^{\prime} n \bar{u}$ id bi-anniyyatihi wa-kānat anniyyatuhu murakkabat («je veux dire, quand l' agent agit (en utilisant) un instrument, il n' agit pas par son essence et son essence n' est pas composée»). Toutefois, le traducteur latin a lu de toute évidence dans son modèle: ánìi íā kāna l-fāil wa-l-maf'ul, leçon attestée dans le manuscrit de Leyde. Cela explique le début de sa traduction, quando agens et factum sunt per instrumentum, à condition de comprendre sunt per dans le sens de «sont mis en rapport». Immédiatement après, non facit per esse suum, peut sous-entendre, comme le fait le texte arabe, agens avant facit, même si ce sous-entendu reste moins évident dans la traduction latine que dans le texte arabe. Quant à la fin de sa traduction, et sunt composita, elle se détourne clairement de l' arabe, sauf si le substantif anniyya avait à faire défaut dans son modèle, ce qui n' est pas le cas dans les trois manuscrits connus. La traduction originale portait-elle alors

49 Taylor 1981, 183,16-184,17.

$50 \quad$ Voir infra, p. 295 .

51 Pattin 1966, p. 179, l. 31 signale une omission après suum, mais celle-ci est conforme aux manuscrits arabes d' Ankara et d' Istanbul. Il est par contre plus étonnant qu' il maintienne la première occurrence de la conjonction et dans le textus, alors que la majorité de ses manuscrits l' omettent en conformité avec le texte arabe ; en plus, l' absence de la conjonction dans la traduction latine est confirmée par les traductions latino-hébraïques, voir Rothschild 1994, p. 462, où on lit dans la traduction française reposant principalement sur le texte de Juda Romano: «... par un instrument, [l' agent] n' agit pas... » (hébr. hēm bi-kelī $\bar{e} y n \bar{o}$ pō $\left.{ }^{e} l\right)$; la traduction plus tardive de 'Eli Habilio comporte en revanche la conjonction: hū' be-'emșa ūt kelì we-lo'yĭf'al (Rothschild 2013, p. 341). La traduction française du Liber de causis (voir Magnard et alii 1990, p. 71), ainsi que la traduction allemande (voir Fidora, Niederberger, 2001, p. 105) maintiennent la lecture de Pattin, mais suppléent, sans l' indiquer, «agent» (Bewirkendes) avant leur traduction du verbe agit. 
et est esse suum compositum, ou tout simplement: et est compositum, en supposant que le traducteur ait estimé redondante l' addition d'esse suum? Mais n' est-ce pas là modifier de façon (purement) hypothétique la traduction latine en fonction du texte arabe? Certes, mais ce n' est qu' après examen de tous les manuscrits qu' il sera possible de décider si le traducteur a trouvé une omission dans son modèle, ou si l'omission s' est produite lors de la transmission de la traduction latine et a pu donner lieu au changement du singulier du verbe est, en son pluriel sunt, après l'omission d' esse suum.

\section{4}

\section{Le latin médiéval}

Notre traduction appartient au douzième siècle; il n' est donc pas exclu que certains mots soient compris dans un sens typique au latin médiéval ou soient écrits selon une graphie spécifiquement médiévale.

En ce qui concerne la graphie, nous n'avons détecté qu'un cas qui mérite une attention particulière. Il s'agit de (causa) exemplaria pour (causa) exemplaris (XIII[XIV], 119,29). Dans la tradition manuscrite, les deux formes exemplaria et exemplaris sont attestées avec, à première vue, une légère majorité de cas portant la dernière forme, majorité parmi laquelle se trouve le manuscrit d'Aoste, Seminario maggiore 71 - mais pas le manuscrit Vat. Ottob. Lat. 1415. On voit mal pour quelle raison, si exemplaris se trouvait dans l'archétype, un copiste l'aurait modifié en exemplaria, qui est une forme extrêmement rare. Nous n' avons pas trouvé la graphie exemplarius comme alternative pour l'adjectif exemplaris dans les dictionnaires de latin médiéval à notre disposition, sauf sous une forme substantivée signifiant alors «copiste ${ }^{52}$. Toutefois, Pattin avait déjà signalé que cette graphie était attestée dans le Thesaurus linguae latinae, où se trouve une référence unique empruntée au Corpus inscriptionum Latinarum $(C I L)^{53}$. En outre, la leçon (causa) exemplaria est présente dans le commentaire de Gilles de Rome, qui cite littéralement le passage: hoc ergo... causa exemplaria, pour ajouter immédiatement après idest causa exemplaris ${ }^{54}$. Cette dernière spécification montre que Gilles est conscient du

\footnotetext{
$52 \quad$ Latham et alii 1975-2013, p. 840.

53 Pattin 1966, p. 165 , note $m$; Thesaurus linguae latinae, tome $\mathrm{v}_{2}$, col. 1326 , avec référence à $C I L$ VIII, 26622. Ce dernier présente une inscription sur une fontaine dans un jardin à Thygga (Dougga-Tunisie), mais l'honnêteté oblige à reconnaître que le mot exemplarius (patrono exemplario) résulte d'un essai de reconstruction du texte, car le monument ne porte pas davantage que patro (espace blanc) plario.

Gilles de Rome, Super Librum de causis, f. 46rG. Il est étonnant que dans la citation du para-
} 
caractère inhabituel de cette graphie, car il sent le besoin de dire explicitement qu'il y a identité de sens parfaite entre cette graphie et celle, plus habituelle, d'exemplaris. Plus frappant encore est le fait qu'il ne considère pas cette graphie comme fautive, ce qui aurait simplement requis une correction en exemplaris. Nous inclinons à croire que la lectio difficilior (causa) exemplaria figurait dans l'exemplaire du traducteur et a été corrigée ensuite par un copiste en exemplaris, qui aurait quant à lui cru à une erreur de copiste dans le modèle (erreur de copiste qui se serait produite à cause d' une attraction avec le $a$ final du mot causa qui précède).

En III, 33,17, le texte latin selon l'édition Pattin porte stramentum. Ce choix peut surprendre car ce terme n' est présent que dans deux des dix manuscrits témoins. Mais l'important manuscrit Vat. Ottob. Lat. 1415 est l'un de ces deux témoins ${ }^{55}$; en outre, le mot figure dans les commentaires d'Albert le Grand, Thomas d'Aquin et Gilles de Rome ${ }^{56}$. En latin classique ce mot signifie «ce dont on jonche le sol» ou encore « couverture»; en latin médiéval, il peut avoir le sens plus précis de tapis (de prière), ce qui correspond plus ou moins au mot arabe bisāt présent dans le texte arabe tel qu' il est conservé dans les manuscrits d'Ankara et d'Istanbul ${ }^{57}$. La remarque de Gilles de Rome dans son commen-

graphe qui commence la Secunda pars principalis commenti, l'édition de la Renaissance porte exemplaris.

L' autre manuscrit reconnu comme important, à savoir celui d'Aoste, propose aussi cette leçon, voir Taylor 1989, p. 89, no. 12. Notons que Vansteenkiste 1967, p. 62-63 avait déjà indiqué qu'il fallait supprimer les crochets que Pattin avait mis autour de stramentum. Mais Vansteenkiste ne dit rien à propos du sens précis de ce terme dans ce cas-ci. De plus, il se base sur l' édition de Bardenhewer pour établir un lien avec le mot arabe bisāt. Cependant, il ne mentionne pas le fait que Bardenhewer a opté pour ce choix en se basant sur la traduction latine. De plus, dans son petit lexique arabo-latin, il rapporte la racine $b s t$ (p. 70 , sub 6) à trois mots latins très différents: stramentum, expansus et simplex, mais oublie de préciser que stramentum correspond à l' arabe bisāt ; expansus à mabsūt et simplex à basitt (qui, à son tour, rend le grec $\dot{\alpha} \pi \lambda \mathbf{o v}_{\varsigma}$ ). Suite à cette confusion due à l' approche peu rigoureuse de Vansteenkiste, Rothschild 1994, p. 418, note 10 (aussi Rothschild 2013, p. 75), a critiqué à tort - comme le montre notre exposé - le choix de la leçon stramentum. Rothschild considère sans doute que le fait que les traductions latino-hébraiques témoignent toutes de la leçon "instrumentum", vient renforcer sa critique. Il est toutefois regrettable qu' il ignore la présence de bisăț dans deux des trois manuscrits arabes connus (et dans un des témoins indirects arabes, voir infra, note 57). Par contre, il explique très bien comment un copiste a pu lire facilement sicut instrumentum au lieu de sicut $(i)$ stramentum.

56 Albert le Grand, De causis et processu universitatis, p. 8o, 1. 24 et p. 87, l. 48; Thomas d'Aquin, Expositio super Librum de causis, p. 23, l. 11; Gilles de Rome, Super Librum de causis, f. 12v.

57 Pour ce sens en latin médiéval, voir Mediae Latinitatis Lexicon, p. 994; pour «tapis (éten$\mathrm{du}) »$ comme traduisant bisāt, voir Dictionnaire arabe-français, t. I, p. 126. Observons encore que la leçon bisāt n' est pas seulement celle des manuscrits d'Ankara et d' Istanbul, 
taire nous semble pourtant d'une signification capitale lorsqu'il note, après avoir cité le bout de phrase (causavit animam): «posuit eam sicut stramentum: aliqui textus habent instrumentum sed melior littera est que dicit stramentum ». Gilles a accès à plusieurs, en tout cas au moins deux manuscrits, où il a pu lire tantôt stramentum, tantôt instrumentum ${ }^{58}$. Sans hésitation aucune, il opte pour la première de ses lectures et indique que cela implique que la Cause première stravit eam [à savoir, l'âme] operationi intelligentiae. Par ailleurs, la leçon stramentum est confirmée dans le commentaire de Thomas d'Aquin qui offre une explication identique à celle de Gilles, la seule différence étant que Gilles utilise le verbe stravit, alors que Thomas opte pour substravit. Mais les deux verbes ont fondamentalement le même sens ${ }^{59}$. Dans le contexte philosophique du Liber de causis, le mot paraît donc avoir plutôt le sens de «substrat», de quelque chose qui sert de «fondement», or c'est un sens attesté en latin médiéval. ${ }^{60}$

Une compréhension spécifiquement médiévale du verbe sequi est sans doute en jeu en I, 3,8 (sequitur). Richard Taylor a déjà bien expliqué que le verbe arabe waliya - dont la signification usuelle est «suivre» - a ici le sens de «être adjacent à» et opte en conséquence pour une lecture en latin (lignes 9-10) causa universalis secunda quae sequitur ipsum [à savoir, le causatum] au lieu de: causa universalis secunda quae sequitur ipsam [à savoir, la causa prima], lecture présente dans l'édition Pattin ${ }^{61}$. Mais n' est-ce pas imposer au verbe latin un sens qu'il n' a nullement? Jean-Pierre Rothschild fait état d'une traduction malencontreuse et inexacte, dont les traducteurs hébraïques ne sont pas venus à bout ou dont ils ont escamoté la difficulté62. Par ailleurs, dans les commentaires d'Albert le Grand, Guillaume de Leus et Johannes Wenck figure la leçon sequitur ipsam ${ }^{63}$, conformément à la majorité des manuscrits témoins de l'édition Pattin ${ }^{64}$. Néanmoins, la consultation d'une trentaine de manuscrits nous a révélé qu' un peu plus de la moitié d'entre eux témoignent

mais elle est confirmée, en outre, par le Kitāb al-fușūl fíl-ma'ālim d' al-'Āmirī (voir Wakelnig 2006, p. 182).

58 Gilles de Rome, Super Librum de causis, f. $12 v$.

59 Thomas d'Aquin, Expositio super Librum de causis, p. 23.

6o Fuchs et alii 1970-2005, vol. vII, p. S 788. Magnard et alii 1990, p. 43, traduit stramentum par «une assise (pour)», alors que Fidora, Niederberger 2001, p. 43, la rendent par Unterlage. Il se pourrait que la compréhension philosophique au moyen âge du mot stramentum comme «substrat» ait eu son origine dans le passage sous discussion.

61 Taylor 1978, spécialement p. 170-172.

62 Rothschild 2013, p. 74.

63 Voir respectivement Albert le Grand, De causis et processu universitatis, p. 67, l. 63; Carron 2016, p. 531, l. 24; Meliadò 2016, p. 262, l. 8.

64 Pattin 1966, p. 134, note 8 indique que seulement deux des dix manuscrits consultés par lui 
d' une leçon ipsum, ce qui est une indication sérieuse en faveur de l'hypothèse selon laquelle cette lectio difficilior était effectivement celle du traducteur luimême. Comme l' a observé Taylor, la variante ipsam a toutes les chances d'être le fruit de l'intervention d'un copiste, qui a constaté une contradiction inhérente à l' affirmation, en comprenant le verbe sequitur dans son sens ordinaire de «suit». Mais le verbe latin peut-il avoir une autre sens? Cette idée est loin d'être évidente si l'on consulte les dictionnaires de latin classique et de latin médiéval. Néanmoins, nous avons trouvé dans le Mediae Latinitatis Lexicon Minus un sens qui n' inclut pas l'idée d'une suite successive, à savoir «se rattacher à ${ }^{65}$. Si ce sens n' exprime pas exactement l'idée de «être adjacent à», il évite néanmoins l'idée d' une «suite». On ne peut donc nullement exclure que Gérard de Crémone, comme traducteur latin, ait donné ce sens, ou un sens qui lui soit proche, au verbe sequi à l' intérieur de ce contexte.

Un dernier cas qui mérite l'attention est l'utilisation du verbe excusare en I, 4,11 comme traduction du verbe istagnna ('an), «être capable de se dispenser (de quelque chose)», «ne pas avoir besoin (de quelque chose)», incluant donc l'idée d' «être suffisant à soi-même». Nous avons cherché en vain un tel sens dans un dictionnaire de latin. Rothschild, conscient du problème, signale que le verbe latin, pourrait avoir signifié ici, au vu de l'arabe, «être exempt, dispensé ${ }^{66}$. De façon surprenante, un sens semblable prévaut dans la traduction de certaines parties des livres naturels du Shifä' d'Avicenne effectuée à Burgos entre 1275 et $128 \circ^{67}$. D' autre part, dans la traduction du De anima de ce même ouvrage, réalisée un siècle plutôt à Tolède par Gundissalinus et Avendauth, le verbe excusare traduit à deux reprises l'arabe kafāa qui peut signifier «avoir quelqu' un exempt de ${ }^{68}$. Notons enfin que dans le Flos Alfarabii (traduction partielle des 'Uyūn al-masāil généralement attribuée à Gun-

portent ipsum. Cela n' est pas correct, car au moins un des huit autres manuscrits, à savoir Bruges, Bibliothèque de la ville 463 (ms A de Pattin), a cette leçon.

65 Mediae Latinitatis Lexicon, p. 96o.

66 Rothschild 2013, p. 76.

67 Avicenna latinus 1987, p. 4, l. 49: excusatum est a (probatione) dans un contexte où il est affirmé que ce qui est évident est exempt de preuve; p. 127, l. 81: excusat (dicere istam dubitationem), à savoir qu' on est dispensé d'exposer le doute évoqué auparavant; et Avicenna latinus 1989, p. 75, 1: (nos) excusat a (reiteratione), signifiant ici «nous exempt de répéter» (dans les trois cas, le texte arabe porte yugnn̄ ['an]). Pour la datation de cette traduction, voir Avicenna latinus 1987 , p. $67^{*}-68^{*}$.

68 Avicenna latinus 1968, p. 183, 94: excusat (nos ab hoc labore), signifiant ici «nous exempte de ce fardeau», et Avicenna latinus 1972, p. 234, 1. 83: excusat (laborem egrediendi spiritum ad aerem), ce qui veut dire en contexte «dispense le pneuma de la peine de se mouvoir vers l' air». Pour la datation de cette traduction, voir Avicenna latinus 1972, p. $92^{*}-95^{*}$; en ce qui concerne les noms de ces traducteurs, voir ibid., p. $98^{*}-103^{*}$. 
dissalinus) on trouve et non excusatio esse eius a causa pour traduire fa-la ganiya bi-wuḡüdihi 'an al-illa, «car il [= le possible] ne peut pas être exempt de cause quand il existe» ${ }^{69}$. Sur base de ces constatations, il paraît donc raisonnable de penser que le verbe excusare pouvait, dès la seconde moitié du douzième siècle, avoir le sens de «dispenser», « exempter». Reste par contre le problème de savoir s'il faut lire avec l'édition Pattin: excusat, ou s'il faut préférer, comme le suggèrent Vansteenkiste et Rothschild, la variante excusa$t_{u r}{ }^{70}$. Les deux formes sont attestées dans la tradition manuscrite, mais, sur la base d'un survol d'une trentaine de manuscrits, la forme passive s' avère la plus établie. Elle est en plus présente dans les commentaires de Guillaume de Leus et Johannes Wenck ${ }^{71}$. Enfin, elle semble être confirmée par les trois traductions latino-hébraïques ${ }^{72}$. Par contre, un bon nombre de manuscrits, dont l'Ottobonianus et celui d'Aoste, portent la forme active, qui a très vraisemblablement constitué la base de la traduction latino-hébraïque de Ḥabilio. Il n' est donc pas aisé de trancher. Certes, on peut admettre avec Rothschild que la forme passive semble préférable d'un point de vue grammatical, mais est-ce suffisant? Enfin, il existe encore un problème additionnel, car on trouve une troisième variante, à savoir excludit, attestée au moins dans le manuscrit St. Gallen, Stiftsbibl., Cod. Sang. 837, f. 22. Le même verbe, au passif cette fois, se retrouve dans le commentaire de Gilles de Rome, f. 2 rK, qui explique la citation «non excluditur ipsius [i.e., causae secundae] actio a causa prima quae est supra ipsam» de la façon suivante: «quasi dicat quod actio causae secundae non excludit immo praesupponit actionem causae primae». Il semble que Gilles trouve la formulation de la traduction latine inhabituelle et sente par conséquent le besoin de la reformuler (quasi dicat) dans un latin plus ordinaire. Il est pourtant frappant de voir qu' il ne mentionne pas la leçon excusat / excusatur, ce qui indique qu'il ne l'a nulle part vue dans la

69 Cruz Hernández 1951, p. 308, l. 40 (arabe) et p. 317, l. 14-15 (latin).

$70 \quad$ Vansteenkiste 1967, p. 78 ; Rothschild 1994, p. 408 (note $5^{\mathrm{B}}$ ) et 2013, p. 76.

71 Carron 2016, p. 532, l. 1; Meliadò 2016, p. 262, l. 1. Observons que Pattin 1966, 134, note 11 indique de façon injustifiée que le commentaire de Guillaume porte la variante excluditur. En fait, celle-ci est présente dans le commentaire de Gilles de Rome (voir la suite de l'exposé).

72 Rothschild 2013, p. 292-293. Hillel: lo’ tippāšêr pe'ullat ha-ri'šōnāh, «l' action de la première ne se dissout pas»; Juda Romano: lo’ tūsar pe'ullat ha-ri'šōnāh, «l' action de la première n' est pas ôtée»; 'Eli Ḥabilio: lo’ yeḥdal 'im zeh po'al ha-sibbāh ha-ri’sōnāh, «l' action de la cause première ne cesse pas pour autant». La première et la troisième de ces traductions semblent reposer plutôt sur excusatur, la seconde pourrait se justifier aussi à partir d'excluditur. M. Rothschild nous signale que, par suite d' une erreur de renvoi au texte, les citations de l'hébreu dans son article de 1994, p. 407, n. 5 et dans celui de 2013, p. 76 sont inadéquates. 
documentation à sa disposition, qui impliquait l' accès à au moins deux manuscrits, comme nous l' avons déjà montré73.

\section{$5 \quad$ Complexité de la transmission de la traduction}

Dans ce qui suit, nous ne discuterons pas tous les passages qui risquent de soulever des difficultés sérieuses à tout qui se voudrait le futur éditeur d'une nouvelle édition critique. Nous nous bornerons à discuter, à titre d' exemple, trois cas à propos desquels Taylor a proposé une autre lecture que celle de l'édition Pattin.

(1) En I, 17,59-62, on lit dans l'édition Pattin: «Quod est quia causa secunda quando facit rem, influit causa prima quae est supra eam super illam rem de virtute sua, quare adhaerit ei adhaerentia vehementi et servat eam ». Taylor propose, sur la base de l'arabe fa-tulzima d̄älika l-shay'a luzūman šadìdan qui signifie «en sorte qu'elle [= la première cause) adhère à cette chose», ainsi qu'en tenant compte de plusieurs manuscrits parmi lesquels Aoste et Vatican Ottobonianus, de lire le segment de phrase en italiques de la manière suivante: «quare adhaerit illud rei adhaerentia vehementi ${ }^{74}$. Cette lecture trouve un appui indirect dans le commentaire d'Albert le Grand, car elle a infixum adhaerat rei, ce qui indique qu'Albert avait illud dans son modèle et l'a interprété comme infixum ${ }^{75}$. D' autres commentateurs, comme Gilles de Rome, Guillaume de Leus et Johannes Wenk confirment par contre la leçon $e i$, mais tant Gilles que Guillaume comprennent ce pronom comme faisant référence à la cause seconde, et non à la chose produite par celle-ci ${ }^{76}$. Cette position a de quoi surprendre, car il semble plus naturel de comprendre le pronom $e i$

73 Voir supra, p. 293.

74 Fidora, Niederberger 2001, p. 38 adoptent cette variante en latin, mais ils traduisent (ibid., p. 39): «weshalb sie jenem Ding stärker anhaftet», ce qui présuppose clairement une leçon illi rei, non illud rei.

75 Albert le Grand, De causis et processu universitatis, p. 68, 1. 33-34. En interprétant ainsi illud, et non en termes de l' action divine ni encore moins de Dieu comme cause première lui-même, Albert écarte complètement toute connotation panthéiste, voire panenthéiste. Selon Pattin (Pattin 1966, p. 137, note 61), le commentaire de Bacon porterait aussi de la leçon illud rei, mais nous n'avons pas trouvé un seul passage qui en témoignerait. Par contre, la leçon est effectivement présente dans l'édition du Liber de causis qui suit celle du commentaire, voir Roger Bacon, Quaestiones supra Librum de causis, p. 162, 1. 15.

76 Gilles de Rome, Super Librum de causis, f. 5vHH; Carron 2016, p. 537, l. 6; Meliadò 2016, p. 264 , l. 9 . 
comme relaté à illam rem qui précède, donc en accord avec l' arabe: à la chose produite par la cause seconde ${ }^{77}$. C' est ainsi qu' au moins deux des traductions latino-hébraïques l' ont compris, lisant sans doute dans leur Vorlage: illi rei ${ }^{78}$. Quand on regarde l' apparat de l' édition Pattin, on a l'impression que les deux variantes figurent dans un nombre presque égal des manuscrits. Un sondage effectué dans une trentaine de manuscrits nous a révélé qu' il n' en est pas ainsi. En fait, un peu plus de la moitié de ces manuscrits portent la leçon illud rei et seulement un cinquième $e i$. N' est-ce pas là une indication sérieuse en faveur de la proposition de Taylor de remplacer cette dernière par la première? Et cela d' autant plus qu' elle correspond à une lecture de l'arabe fa-yulzima (éd. Taylor: fa-tulzima) dālika l-shay’a luzūman šadīdan, attestée dans les manuscrits d'Ankara et d' Istanbul ${ }^{79}$, où dea lika figure comme sujet de la phrase. Cette piste écarte pourtant complètement le problème que soulève de toute évidence cette leçon. En effet, à quoi le pronom illud / dālika se réfère-t-il? La réponse est sans doute: «le fait que la cause première, qui est au-dessus de la cause seconde, influe sur la chose, produite par celle-ci, de sa propre puissance», bref, l' action d'influer de la cause première. Si cette lecture n' est pas entièrement dépourvue de sens, elle ne tient pas compte de l' affirmation qui vient tout de suite après, selon laquelle c' est plutôt la cause lointaine elle-même, et non son action, qui continue à adhérer à la chose sur laquelle elle influe sa puissance, et qu'elle conserve après que la cause prochaine s' en soit séparée: «Iam ergo manifestum est et planum quod causa longinqua est vehementius causa rei (...), et quod ipsa influit virtutem suam super et servat eam (...), immo remanet in ea et adhaeret ei adhaerentia vehementi, secundum quod ostendimus et exposuimus».

En outre, d'un point de vue purement grammatical, tant en arabe qu' en latin, il est loin d'être évident que ce pronom démonstratif puisse se référer

77 Il en va ainsi de la traduction française, basée sur une lecture du latin $e i$ : «La raison en est que lorsque la cause seconde produit une chose, la cause première, qui est au-dessus d'elle, influe sur cette chose de par sa propre puissance. C' est pourquoi elle y [faisant référence sans ambiguïté aucune à 'cette chose'] adhère d'une adhérence plus forte et la conserve» (Magnard et al. 1990, p. 41).

78 Rothschild 1994, p. 406, traduit l'hébreu de Juda Romano: «et [sous-entendu: la cause première] y (= cette chose, produite par la cause seconde et recevant l'influence de la cause première) adhère»; mais Juda Romano explicite: tiddebaq 'im òtō ha-dābār, « elle adhère à cette même chose»; de même 'Eli Ḥabilio: hỉ mehubberet la-dābār ha-hü', « elle est attachée à la chose en question »; Hillel seul emploie des pronoms dont les référents sont incertains, lisant probablement ei (Rothschild 2013, p. 294-295).

79 En l'absence de points diacritiques, comme c'était souvent le cas dans les manuscrits anciens, la graphie des deux formes du verbe est identique et les deux lectures sont donc également possibles. Taylor 1981, p. 142, note 39-40 remarque que les manuscrits d'Ankara et d'Istanbul (qui ne sont pas datés, mais relativement tardifs) portent la vocalisation $y u$. 
à l' ensemble de la phrase qui précède. On s' attend plutôt à ce qu' il soit lié avec le mot qui suit, qui est rei / l-shay ${ }^{\prime}{ }^{80}$. Dans ce sens, l' utilisation du pronom ei se révèle plus adéquate, car il réfère à «cette chose-là», nonobstant son identification dans certains commentaires avec la cause seconde, comme nous l' avons indiqué plus haut. Toutefois, on ne peut pas ignorer l'attestation massive de la formulation illud rei. Pourrait-on imaginer à sa base une lecture fautive d'illi rei? Comme nous avons découvert cette dernière leçon dans trois manuscrits, à savoir, Césène, Bibl. Malatestiana, Plut. XXIII, Dext. 6; Vienne, ÖNB 2491 et Angers, вм $45^{81}$, cette hypothèse gagne en probabilité, d'autant plus que le «i» d'illi est pourvu d'un petit accent, comme d'ailleurs aussi le «i rei qui suit. Un copiste pourrait avoir lu ce «i avec accent» comme un « $\mathrm{d}$ » et avoir transformé ainsi illi en illud. Mais cela n' est pas à notre avis la seule explication possible. En effet, s' il ne fait pas de doute que la leçon illi rei fut présente dans l' archétype, car elle rend l' arabe de façon parfaite, on ne peut pourtant pas exclure qu' illud rei y figurait comme traduction alternative, dans la mesure où le traducteur hésitait entre un pronom démonstratif lié à un substantif ou un sujet de la phrase où il est question d' adhérence ${ }^{82}$. En somme, on se trouve devant un choix compliqué quant à ce qu'il faudrait mettre dans le textus d'une éventuelle nouvelle édition critique: (1) illi rei, en fonction de la compréhension la plus évidente du texte l'arabe, qui paraît aussi avoir à son crédit l'hébreu de deux des traducteurs, à moins qu' elle ne procède que d'un effort d'éclaircissement de la part de ceux-ci; ou (2) illud rei, en tenant compte de ce dont témoignent la majorité des manuscrits; ou encore (3) tout simplement $e i$, sur la base de la tradition des commentaires, mais, en même temps, d'une minorité importante de manuscrits et d' une des traductions latino-hébraïques.

(2) En XI(XII), 107,79, on lit dans l'édition Pattin: agens in rem, à corriger selon Taylor en agens (ou, éventuellement, agens vel quae est) in re, car le texte arabe porte: (al-shay') al-kāin fíl-shay', «(la chose) qui est dans la chose ${ }^{83}$. Dans la partie de la tradition manuscrite que nous avons examinée, nous avons découvert que la vaste majorité porte ou bien agens in rem, ou bien agens in re, sans qu' une nette préférence pour une de ces deux variantes ne se dessine, ce

8o La traduction anglaise que Taylor (voir Taylor 1981, p. 285) propose du texte arabe, à savoir: «strongly adheres to that thing», en constitue une illustration parfaite.

81 En outre, il est à noter que le manuscrit St. Gallen, Stiftsbibl., Cod. Sang. 837 présente illi sans l'addition rei.

82 Ce problème se posait certainement si le traducteur, dans son modèle, lisait fa-yulzima, car dans le texte arabe aucun substantif masculin ne précède à l'intérieur de la même affirmation; cette observation est à l' origine de la vocalisation $t u$ (lzima) dans l'édition du texte arabe de Taylor (Taylor 1981, p. 351-352).

83 Taylor 1989, p. 94, notes 45-46. 
qui ne correspond pas vraiment à ce que l' apparat de Pattin laisse croire. Les commentaires de Gilles de Rome et de Thomas d'Aquin suivent clairement la première leçon, tandis que les traditions latino-hébraïques avaient pour base un modèle où figurait la dernière ${ }^{84}$. Si on veut rester le plus proche du texte original arabe, il est clair que la dernière variante doit être retenue pour l'édition, mais si on tient compte de la réception de la traduction latine dans les commentaires, on ne peut pas ignorer la première sans plus d'égards. Selon nous, il ne faudra pas uniquement la signaler dans l' apparat des variantes, mais également insister sur son importance particulière d' une façon ou d'une autre ${ }^{85}$. Reste le fait qu'agens a de quoi surprendre comme traduction de $k a \bar{\imath} i n$. Même si on le comprend dans le sens de «étant actif dans», qui est un de ses sens possibles en latin médiéval ${ }^{86}$, il s' agit toujours d'une traduction interprétative ${ }^{87}$. Il n'est donc pas sans intérêt de constater que dans quatre des dix manuscrits témoins de l'édition Pattin, la leçon agens vel quae est in re est attestée. Il s' agit sans doute d'un essai de double traduction, où la traduction quae est constitue une traduction assez littérale, alors que la traduction agens se présente davantage comme une traduction libre, inspirée en toute probabilité par la qualification per modum causae qui suit ${ }^{88}$. En outre, plusieurs manuscrits, par exemple Vat. Urb. lat. 206, Assise, BC 298 et Leipzig, Universitätsbibliothek 1382, témoignent de la variante: agens secundum quod est. La formule res agens vel quae est in re a-t-elle été jugée peu claire par un copiste et a-t-il opté pour une correction ad sensum en remplaçant quae est par secundum quod?

84 Pour les premières, voir Gilles de Rome, Super Librum de causis, f. 41rH et Thomas d' Aquin, Expositio super Librum de causis, p. 80, l. 26 (secundum quod causa agit in effectum); concernant les traductions latino-hébraïques, voir Rothschild 1994, p. 445: «(la chose) qui agit (comme cause) dans une autre (...) n' est en elle (...)» (ha-dābār ha-pōél be-dābār [...] èynō/ēynennū $b \bar{o}[\ldots])$, chez Juda Romano et chez 'Eli Ḥabilio; Hillel de Vérone omet tout le développement de cette proposition (Rothschild 2013, p. 320-321).

85 Même si tout laisse croire que la variante agens in rem résulte de l' initiative d'un copiste, qui comprenait agens in dans son sens obvie, à savoir «agissant sur», et a par conséquent choisi de mettre ce qui en constitue l' objet à l' accusatif au lieu de l'ablatif, nous serions tentés de mentionner les deux variantes dans le textus (mais en indiquant la deuxième avec un astérisque), car celle-ci a clairement influencé la compréhension de plusieurs commentateurs latins.

86 Prinz et alii 1959 sqq., vol. 1, p. 401.

87 Dans les lexiques publiés de l' Avicenne latin, nous avons trouvé un seul endroit où le verbe kāna a été rendu en termes d' «agere», à savoir dans le De anima (voir Avicenna latinus 1968, p. 30, l. 3), mais c' est à l' intérieur d'une phrase dont la traduction latine dévie sensiblement de l' arabe (voir Avicenna latinus 1968, p. 30, note 2-3).

88 Outre les quatre manuscrits auxquels Pattin se réfère, on trouve la même variante dans le manuscrit d' Angers, BM 450. 
D' autres variantes significatives de cette formule se trouvent dans (au moins) deux manuscrits: Paris, BnF 6569, qui porte agens in rem (mais avec la lettre 'm' exponctuée) sur la ligne, et quae est au-dessus de la ligne; et Chicago, Vault Case 23, qui présente la formulation complexe agens in rem vel quae est in re. Le manuscrit de Chicago pourrait témoigner de ce que son copiste ait observé l'existence de la double traduction, mais aussi celle de la variante agens in rem, et ait alors estimé qu' en offrant la formule qui est la sienne, il articulait la vraie alternative telle que la traduction originale l' avait exprimée. Ou bien l' archétype de la traduction portait-il vraiment agens in rem, le traducteur ayant, après révision, exponctué le $m$ d' in rem et ajouté au-dessus de la ligne $e t$ quae est, comme peut le suggérer le manuscrit de Paris? En absence d' un survol complet de tous les manuscrits, il est prématuré de décider, mais tout indique néanmoins que la traduction latine portait à l' origine, ou sinon très tôt lors de sa transmission, un essai de double traduction. Ce que Taylor avait quelque part pressenti, se révèle de plus en plus une quasi-certitude.

En XVIII(XIX), 150,75-77, Pattin présente la lecture suivante: «Et hoc non fit ita nisi quoniam est ipsa $\langle\ldots\rangle$ neque intellectualis tota neque animalis tota neque corporea tota, neque pendet per causam (...)». Taylor, de son côté, propose de lire: «Et hoc non fit ita nisi quoniam est ipsa expositio neque intellectualis tota neque animalis tota neque corporea tota, neque [Taylor 1981: non] pendet per causam (...) $\gg^{89}$. En effet, le mot expositio est attesté dans les manuscrits Oxford, Bodleian Selden sup. 24 (comme Pattin l'avait déjà signalé) et Aoste; il constitue une traduction qui, bien que ne convenant pas au contexte, rend littéralement le terme arabe šarh, présent dans les trois manuscrits témoins du texte arabe ${ }^{90}$. De surcroît, on peut invoquer deux témoins indirects en faveur d' une mention explicite de ce mot: le commentaire d' Albert le Grand, ainsi que la traduction latino-hébraïque de Juda ${ }^{91}$. Malheureusement, Taylor a perdu de vue que les mots est ipsa, ainsi que la conjonction neque avant

89 Taylor 1989, p. 97, note 58. Concernant la proposition de lire non, voir Taylor 1981, p. 485, note 136 ; cette note n' est pas reprise dans son article de 1989, sans doute parce qu'il s' est aperçu entretemps qu'une telle lecture pose grammaticalement problème.

9o La leçon šarğ retenue par Bardenhewer (Bardenhewer 1882, p. 94, l. 1) résulte d'une correction d' auteur (voir l'apparat l. 1); il nous paraît difficile de considérer ce mot comme un équivalent possible du grec genos, comme Rothschild (Rothschild 1994, p. 460, note 6) le suggère.

91 Pour le premier, voir Albert le Grand, De causis et processu universitatis, p. 155, l. 10-18: «Et huius quidem causa non est alia nisi quia ipsa anima secundum quod anima est, secundum totum suum esse, nec est expositio sive expressio sive sigillatio intelligentiae, ut tota scilicet intellectualis (...). Et anima quidem non pendet per causam quae est supra eam». Pour le second, la traduction est: «parce que toute explication (ba-'abūr [heyōt] kol bè ūu r)», voir Rothschild 1994, p. 46o, note 6; Habilio (... le-mah še-hỉ èynāh śikhlìt kullāh...) traduit 
intellectualis sont absents des deux manuscrits mentionnés et qu'ils portent tous deux à la fin non pendet. Par conséquent, la reconstruction correcte serait: «Et hoc non fit ita nisi quoniam expositio intellectualis tota neque animalis tota neque corporea tota, non pendet per causam (...) $»^{92}$. Mais les manuscrits latins témoignent d'une variété assez surprenante à cet endroit. Nous ne pouvons pas présenter tous les détails dans ces pages, mais pour notre propos il est important de constater que la formule neque pendet est attestée de façon limitée. Selon notre documentation, la plupart des manuscrits comportent non pendet (ou non penderet / non pendent) et le verbe est après quoniam. Toutefois, une grande diversité de variantes viennent après cette dernière conjonction: ipsa intelligibilis, ipsa neque intelligibilis, intellectibilis ipsa, ipsa scilicet anima, intelligibilis tota, intelligibilis atque, ex ipso, dispositio, voire exputo et ex tempore (?).

Ensuite, il est à noter qu' aucun des manuscrits que nous avons consultés (en plus de ceux déjà utilisés par Pattin) ne porte explicitement le mot expositio et que son attestation se limite donc à deux manuscrits sur un ensemble d'une quarantaine, plus le modèle du traducteur hébreu Juda Romano. Toutefois, la variante exputo, dépourvue de sens, qui se lit dans le manuscrit Cologny, Bodmer С в 10, est relativement proche d'expositio et, compte tenu de l' abréviation habituelle de ce dernier, s' explique facilement par une lecture erronée, suite à un manque d'attention porté au contexte. La même explication pourrait être valable pour la variante ex ipso attestée dans plusieurs manuscrits. Par contre, la variante dispositio qui est présente dans les manuscrits Cava de' Tirreni (Salerne), B. ss. Trinità 31, et Genève, BGE, Lat. 76, pourrait témoigner d' un essai de traduction alternative. Nous avons déjà noté qu'expositio est une traduction en soi correcte du mot arabe šarh. Mais il est clair que dans notre texte ce dernier n' est pas utilisé dans son sens habituel. Comme dans les Plotiniana arabica, il signifie plutôt «arrangement», «ordre ${ }^{93}$. Comme il s' agit là d' un des sens possibles du mot dispositio en latin médiéval ${ }^{94}$, on a l'impression que son choix ne dépend pas du hasard, mais d'une initiative délibérée, sans doute du traducteur lui-même qui, ayant saisi que sa traduction littérale expositio posait

littéralement quoniam est ipsa neque intellectibilis tota; Hillel de Vérone omet le développement (Rothschild 2013, p. 336-337).

Nous ne tenons pas compte ici de l'addition (accidentelle?) de ex ea est après corporea dans le manuscrit d'Oxford, Bodl., Selden sup. 24.

93 Voir Badawi 1955, p. 20, note 2; pour un exemple concret, voir, par exemple, Épitre sur la science divine, Badawi 1955, p. 178, 1. 4, où le terme - tout en étant lié par un wa-epéxégétique avec le mot nizām- («ordre»), est traduit: «arrangement» par Geoffrey Lewis (voir Plotini Opera, t. II. Enn. IV-VI, 1959, p. 333).

Voire Latham et alii 1975-2013, p. 692. 
problème, a opté pour une alternative qui rende mieux le sens spécifique et exceptionnel du terme arabe dans ce contexte. Par conséquent, on ne peut nullement exclure la lecture suivante dans l' exemplaire du traducteur: quoniam expositio dispositio (ce dernier mot a peut-être écrit en marge ou supra lineam). Faut-il en déduire que la lecture de l' édition Pattin doit être ignorée? Nous ne le croyons pas. Certes, elle n' est justifiée que par un nombre relativement limité de manuscrits, mais elle trouve un support indirect dans le commentaire de Gilles de Rome et, en outre, dans la traduction latino-hébraïque de Habillo ${ }^{95}$. Elle est donc significative au niveau de l'histoire de la réception. Mais on ne peut pas entièrement exclure qu' elle remonte en fin de compte au traducteur lui-même. La disparité de la tradition manuscrite invite à la prudence. Ce n' est que sur la base d'un solide et précis stemma codicum qu' il sera possible se prononcer à ce propos.

\section{Traductions posant problème en comparaison avec le texte arabe}

À nouveau, nous nous limitons à trois cas exemplaires.

(1) $\operatorname{En} \operatorname{XIX}(\mathrm{Xx}), 158,15^{-17}$, l' édition Pattin porte: «(...) ita quod est bonitas, et bonitas [et virtus] et ens sunt res una. Sicut ergo ens primum et bonitas sunt res una ». Taylor, qui se fonde surl' arabe et sur un nombre important de manuscrits latins, opte pour l'omission au lieu de la suppression de $e t$ virtus $^{96}$. Dans sa thèse, il remarque en outre que la fin de la traduction latine se détourne du texte arabe, où se trouve: fa-ka-mā șārat al-huwiyya

95 Respectivement, Gilles de Rome, Super Librum de causis, f. 64vR-65rR, Rothschild 1994, p. 46 o, note 6 .

96 Taylor 1989, p. 98, note 62. Taylor, dans sa thèse, remarque en plus qu' il comprend l' arabe d' une façon différente de la traduction latine, bien que celle-ci soit en soi possible (Taylor 1981, p. 488, note 144). La différence d'interprétation consiste surtout dans la compréhension de la préposition bi-annahu, que le traducteur latin a rendu par ita quod, alors que Taylor la rend par: «by virtue of». De façon un peu surprenante, la traduction latinohébraïque de Juda Romano porte la leçon be-derekh še-, que Rothschild (Rothschild 1994, p. 462) traduit: «en tant que», ce qui semble plutôt correspondre à une lecture de la traduction latine: inquantum (en raison d' une confusion avec ita quod ou traduction alternative présente dans l'archétype du traducteur, mais nous n'en avons pas encore trouvé confirmation dans la tradition manuscrite latine); Ḥabilio porte ' $i m$, «si », mais la suite est altérée: 'im hāyāh ha-ṭōb we-ha-heyōt ha-nimṣāōt, «si la bonté et l' être sont les existants», soit parce que des mots auraient manqué au modèle latin (ita quod et bonitas et ens una?), soit par une volonté délibérée de clarification, chez un traducteur qui (Rothschild 2013a, p. 64) se pique peut-être d'écriture humaniste; Hillel omet le développement (Rothschild 2013b, p. 338-339). 
l-ülā huwiyyatan wa-hayran naw'an wāhidan («et comme l'être premier devient être et bonté d'une façon singulière»). Il indique, à l'origine de cette non-conformité, soit une corruption dans le modèle du traducteur, soit l'arrivée accidentelle d' une (ou plusieurs) déformation(s) lors de la transmission de la traduction ${ }^{97}$. Il signale d'ailleurs, en faveur de la seconde alternative, la présence du verbe funt au lieu de sunt dans le manuscrit d'Aoste. L'examen des manuscrits à notre disposition nous a révélé que cette dernière leçon n'est pas seulement attestée dans le manuscrit d'Aoste, mais qu' elle est la plus fréquente. Il faut pourtant noter que le copiste du manuscrit Genève, BGE, Lat. 76, a exponctué funt et l' a fait suivre immédiatement sur la même ligne par la forme, dite corrigée, sunt. Cette dernière leçon est aussi celle retenue par Gilles de Rome ${ }^{98}$, et a fonctionné sans doute comme modèle pour les traductions latinohébraïques ${ }^{99}$. Il est difficile de savoir si sunt résulte d'une lecture fautive de fiunt (sans doute inspirée par la phrase qui précède où l'on trouve effectivement la formule sunt una res), ou s'il faut considérer les deux verbes sunt et fiunt comme un essai de double traduction du verbe arabe șāra. Quelle que soit l'option choisie, la traduction latine ne correspond pas directement au texte arabe, mais elle peut être qualifiée de reformulation légèrement interprétative, car elle accentue davantage que ne le fait le texte arabe l'unité foncière entre l'Être premier et la Bonté100.

(2) En XXIII(XXIV), 179,38-39, la traduction latine lit dans l'édition Pattin: «influens vero existens unum non diversum, influit (...)», tandis que le texte arabe porte: wa-ammā al-mufíd fa-innahu wähidun gayr muhtalifin yufidu $(. . .)^{101}$, «mais comme celui qui influe est certes un, sans diversité, il influe (...)». La présence d'existens ne s' explique donc pas aisément, comme l'a remarqué Taylor. Les traductions latino-hébraïques ne semblent pas l' avoir eu dans leur modèle, car elles affirment: «Mais celui

$97 \quad$ Taylor 1981, p. 489, note 146.

98 Gilles de Rome, Super Librum de causis, f. $69 \mathrm{rX}$.

99 Rothschild 1994, p. 462, traduisant Juda Romano: «Ainsi, comme l'Existant premier et le Bien sont une seule chose» (hēm dābār ehâad, chez Ḥabilio comme chez Juda; Hillel omet le développement [Rothschild 2013, p. 338-339]).

100 Observons que le manuscrit Vat. Lat. 14717 porte la lecture: sicut ergo ens primo et bonitas sunt res una, mais on se demande si la leçon primo ne s'est pas produite par attraction avec le mot ergo qui précède (dans les deux cas, le « 0 » étant écrit au-dessus de la ligne).

101 Taylor 1981, p. 241, l. 13-14, mais nous suivons la leçon al-mufiḍ, présente dans le manuscrit de Leyde, au lieu de celle de fayd, que Taylor retient. Contrairement à ce qu'il indique (Taylor 1981, note 13) le latin influens n'équivaut pas à la seconde, mais à la première de ses deux lectures. 
qui s'épanche est un, sans connaître de diversité, épanchant (...) $\gg^{102}$. Elles suggèrent la présence d'un est à sa place. Selon l'apparat de Pattin, le manuscrit Bruges, Bibliothèque de la Ville 463 , porte sic au lieu de vero ${ }^{103}$. Cette leçon semble recevoir une confirmation assez significative dans le manuscrit de Florence, Ashburnham 1674, car le copiste y a écrit sit, mais a exponctué le $t$ et l'a corrigé en $c$ au-dessus de la ligne. Pourtant, deux autres manuscrits pointent dans une autre direction: le ms Berlin, Staatsbibliothek M 1494, Lat. Qu. 449, où se trouve sit, suivi de existens, mais où ce dernier mot a été barré; et le ms Rouen, BM 920, qui porte la leçon sit vel existens. Ils suggèrent que le traducteur a hésité sur la manière de rendre l' arabe et a présenté un essai de double traduction. Mais alors, le conditionnel ne convient pas vraiment. Par contre, la leçon sic nous paraît intéressante dans la mesure où elle permet de mieux se rapprocher la traduction de l'original arabe. En effet, en ponctuant de la façon suivante: «Influens, sic existens unum non diversificatum, influit (...)», on peut comprendre: «l'influent, qui existe ainsi en tant qu'un, non diversifié, influe...», retrouvant ainsi un sens qui ne s'éloigne pas beaucoup de celui du texte arabe. Mais on trouve encore une dernière variante, qui mérite notre attention, à savoir influens vero ens unum (...). Elle est attestée dans le manuscrit Paris, BnF, lat. 16082. À nouveau, en ponctuant influens vero, ens unum (...), «mais l'influent, en étant un (...)», ce qui recoupe largement l' arabe. Des recherches sont clairement requises à l' avenir afin de voir dans quelle mesure chacune de ces variantes est représentée dans la tradition manuscrite, voire même de voir si une autre variante ne peut pas se révéler - par exemple est. Il ne faut cependant jamais perdre de vue que la lecture de l'édition Pattin a une base solide dans la tradition manuscrite et trouve confirmation dans le commentaire de Gilles de Rome ${ }^{104}$.

(3) Enfin, en $\operatorname{xxv}(\mathrm{xxVI}), 188,98-\mathrm{oo}$, on lit chez Pattin: «Si ergo hoc est ita, substantiae stantis per essentiam suam non separatur causa semper»«S'il en est ainsi, la cause de la substance subsistant en elle-même n' est jamais mise à part» ${ }^{105}$. Mais il est dit en arabe: fa-in kāna hād $\bar{a} h \bar{a}-k a \underline{d} \bar{a}$

102 Rothschild 1994, p. 470; textes hébreux de Juda Romano et de 'Eli Habilio, Rothschild 2013,

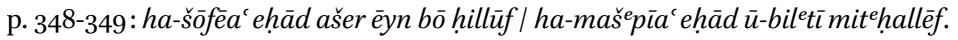

103 La même variante est attestée dans les manuscrits de Troyes 1374 et Bordeaux 421.

104 Gilles de Rome, Expositio super Librum de causis, 1550, f. 83vV et 84rV.

105 Les traductions française (voir Magnard et alii 199o, p. 77), et allemande (voir Fidora, Niederberger 2001, p. 125) contemporaines traduisent ad sensum en affirmant que la cause de la substance subsistant en elle-même n' en est jamais séparée, mais nous ne voyons pas comment le génitif substantie stantis peut se référer à ce qui fait l' objet de l' action de séparer. 
kāna l-ğawhar al-qā̉im bi-d̄âtihi lā yufāriqu 'illatahu abadan ${ }^{106}$, «S' il en est ainsi, la substance qui subsiste en elle-même ne se sépare jamais de sa cause». Toutefois, dans son apparat, Pattin indique que le manuscrit Paris, $\mathrm{BnF} 6318$ propose une autre lecture, à savoir : «(...) substantia stans per essentiam suam non separatur a causa», et que celle-ci fut aussi à l' origine celle du manuscrit Vat. lat. 2089, mais qu' on y trouve en marge une correction de substantia stans en substantiae stantis, ainsi que la suppression de la préposition $a$ avant caus $a^{107}$. Cette dernière correction a de quoi surprendre, car la lecture du manuscrit de Paris, qui est également attestée dans le manuscrit de Leipzig, Universitätsbibliothek 1382, correspond parfaitement à l' arabe. En outre, le commentaire d'Albert le Grand, témoigne d'une même lecture tout en apportant quelques modifications mineures: «Si ergo hoc ita est, substantiae stantes per essentiam suam semper ita se habent quod non separantur a sua causa ». Enfin, les manuscrits de Vienne, ÖNB 2491, et Admont, Cod. 405, témoignent eux aussi d'une leçon substantia stans, mais le premier ne porte pas la préposition $a$ avant causa, tandis que dans le dernier la préposition en question est absente sur la ligne et a été ajoutée juste au-dessus ${ }^{108}$. Tout laisse croire, par conséquent, que la traduction originale portait:«(...) substantia stans per essentiam suam non separatur a causa», et que, sans doute la préposition $a$ a assez rapidement été oubliée par un copiste dans la transmission de la traduction, pour qu' un copiste lise au final causa d'une façon assez naturelle comme un nominatif et modifie le nominatif substantia stans en génitif. Cette dernière lecture est incontestablement la plus attestée dans la tradition manuscrite. Elle est confirmée par Gilles de Rome et la traduction latino-hébraïque de Juda Romano (mais 'Eli Ḥabilio traduit au contraire: «la substance qui subsiste par elle-même ne se sépare pas de sa cause»), même si la notion de «cause» y est placée directement avant celle de «substance qui subsiste en elle-même » ${ }^{109}$.

\footnotetext{
106 Taylor 1981, p. 250, l. 1-2 [Bāb 25, l. 12-13].

107 Pattin 1966, p. 189, notes 98-99. Observons que nous avons constaté la présence (non signalée par Pattin) de la préposition $a$ dans le manuscrit du Vat. lat. 2089.

108 Insistons toutefois sur le fait que le verbe separare peut se construire seulement avec l' ablatif (Gaffiot 200o, p. 1444). D' autre part, il est intéressant de noter qu' au moins deux manuscrits, qui portent la leçon substantiae stantis mentionnent néanmoins la préposition $a$ avant causa.

109 Respectivement, Gilles de Rome, Super Librum de causis, f. 91vX-92rX; Rothschild 1994, p. 473, traduisant d'après Juda Romano; Rothschild 2013, p. 353 (Juda Romano: sibbat ha-'eșem hā-'ōmēd be-'așmō lo’ tippārēd; 'Eli Ḥabilio : ha-'eșem hā-'ómēd be-'așmūtō lo’yippārēd mi-sibbātō).
} 
Sachant que le manuscrit le plus ancien connu du texte arabe est postérieur à la traduction latine, celle-ci n'est pas sans valeur pour l'établissement du texte arabe. Ce point a généralement été reconnu dans la recherche contemporaine. Ce qui en a été dit suffit d' ailleurs, et nous n' avons pas à nous en occuper davantage, car ces remarques dépasseraient largement le cadre de la présente recherche. Néanmoins, nous tenons à attirer l' attention sur un élément qui n'a pas encore reçu toute l' attention qu' il méritait, à savoir la confirmation de certaines leçons de la traduction latine par des témoins indirects du texte arabe. En attendant un examen exhaustif, nous nous limitons à signaler quelques cas exemplaires.

La traduction latine dévie à plusieurs reprises du texte arabe tel qu'il a été transmis par le manuscrit de Leyde, mais correspond à des leçons attestées à la fois dans les manuscrits d'Ankara et d'Istanbul. Ces dernières sont maintes fois confirmées par les témoins indirects arabes. Il en est ainsi pour la mention de simplex en XXVIII(XXIX), 199,52, car le mot arabe qui lui correspond, à savoir mabsüt, est attesté aussi dans le Liber de causis II, ainsi que dans les Questions siciliennes et dans le Budd d' Ibn Sab'īn ${ }^{110}$. Dans le dernier ouvrage, nous avons trouvé plusieurs cas semblables, comme par exemple:l'omission de mumtadd (VI(VII), 69,86/ p. 215, l. 16); et cum aeternitate / wa-ma'a al-dahr (VI(VII), 71,5/ p. 216, l. 3); virtus / quwwa (Ix (x), 94, 18 / p. 201, l. 3); dives / al-ganī ( $\mathrm{xx}(\mathrm{xxI})$, 164,54/ p. 133, l. 25); omission de 'anī ( $\mathrm{xx}(\mathrm{XXI}), 164,59$ / p.134, l.2 ); et bonitas eius est res una / wa-l-hayr šay' wāhid (xx(xxI), 164, 6o-61 / p. 134, l. 3); apud nos / indanā (XXI(XXII), 168,77 / p. 133, l. 16). Dans un cas, ergo intelligentia (XII(XIII), 113,1) est conforme à une leçon $f a-l$-'aql, qui est attestée dans le seul manuscrit d'Ankara parmi les trois témoins manuscrits arabes, mais qui est aussi présente dans le Budd (p. 52, l. 4). Il est clair pour l' ensemble de ces cas, que le traducteur latin respecte le texte tel qu'il le lit dans son modèle. Il n' est donc pas autorisé, pour ces cas, de modifier le texte de la traduction ni d'y intervenir en supprimant des mots ou en signalant des omissions (supposées). Au cas où il serait patent qu'il n'y a pas accord avec le texte arabe original, il faudra en faire état dans une note spéciale.

Mais il y a aussi des cas où les témoins arabes indirects se montrent particulièrement intéressants en présentant des leçons non attestées dans les manus-

110 Liber de causis II, p. 331, l. 10, respectivement Akasoy 2006, p. 406, l. 17 / Spallino 2002, p. 238, l. 8 et Katturah 1978, p. 312, l. 17 . 
crits arabes du Liber de causis, auxquelles la traduction latine correspond pourtant parfaitement. Nous l' illustrerons par trois exemples:

1) proposition $\mathrm{xxx}(\mathrm{xxxI}), 213,66$ : manifestum igitur est quod. Aucun des trois manuscrits arabes ne permet d' expliquer cette traduction, mais dans les Fuṣūl d' al-Amirī se trouve la formule: fa-qad zahara idhan, à laquelle le latin est pleinement conforme.

2) proposition $\mathrm{XXV}(\mathrm{XXVI}), 188,97:$ et servantem eam. Cette traduction trouve un support partiel dans le manuscrit de Leyde, qui porte wa-l-hăfiza $a^{111}$, mais le Liber de causis II et le Budd expliquent en plus la présence de eam en lisant wa-l-hăfiza lahu ${ }^{112}$.

3) proposition VIII(IX), 79,47: omnis intelligentiae fixio. Les trois témoins arabes portent innama $\bar{a}$, «seulement», «ne... que ${ }^{113}$, mais cette expression est absente de la traduction latine; toutefois, l' absence dans la traduction latine de l' expression «seulement», «solummodo» se justifie sur la base de leçon inna, présente dans le Liber de causis $\mathrm{II}^{114}$.

Cette liste n' est sans doute pas exhaustive, mais montre à suffisance que la traduction latine se base parfois sur le témoignage d' une leçon arabe qui n' est pas attestée dans la tradition manuscrite connue du texte, mais uniquement dans ses témoins indirects ${ }^{115}$.

Nous aimerions enfin attirer l'attention sur un cas à propos duquel Bardenhewer a essayé de reconstruire, à partir de la traduction latine, une omission qui prend place vers le début du chapitre 23. Il rend secundum dispositionem à deux reprises par 'alā tartīb $b^{116}$. Se basant sur les manuscrits d'Ankara et Istanbul, Taylor a édité: 'alā ḥăla... 'alā ḥă ${ }^{117}$. Cette dernière leçon est plus ou moins confirmée par la Réfutation du pseudo-Platon et par le Liber de causis II, qui portent: 'alā hâal... 'alā hăall18. Le terme arabe qui correspond au mot latin dispositio est donc hạal, non pas tartībl19.

\footnotetext{
111 Taylor 1981, p. 502, note 182.

112 Voir Liber de causis II, p. 331, l. 4-5 et Katturah 1978, p. 134, l. 14.

113 Taylor 1981, p. 175, l. 2.

114 Liber de causis II, p. 343, l. 13 .

115 Ce témoignage de leçons communes entre traduction latine et témoins indirect arabes, sans préjuger de sa valeur exacte, mérite incontestablement une attention sérieuse et détaillée en vue de l'édition critique du texte arabe.

116 Bardenhewer 1882, p. 102, l. 2-3.

117 Taylor 1981, p. 338, l. 3-4.

118 Türker 1965, p. 58, § 2, l. 1-2 / Badawi 1974, p. 338, l. 12-13, respectivement Liber de causis II, p. 331, l. 14-15.

119 Nous signalons ce cas parce que la reconstruction hypothétique de Bardenhewer a induit Rothschild en erreur (Rothschild 1994, p. 470, note 1).
} 


\section{Traduction latine et le témoignage de la traduction hébraïque de Zerahyah}

La traduction hébraïque de Zerahyah, qui pourrait se baser à la fois sur le texte arabe et la traduction latine ${ }^{120}$, constitue clairement un témoin que ni l'éditeur du texte arabe, ni celui de sa traduction latine ne peuvent ignorer, comme il ressort clairement de l' article de 1994 de J.-P. Rothschild. Nous ne pouvons pas ici en évoquer tout le détail, mais nous essayerons à travers quelques cas exemplaires de démontrer son intérêt en vue d'une édition critique tant du texte arabe - du moins quand il est conforme avec le manuscrit de Leyde contre le latin - que de la traduction latine.

Il faut d' abord observer que la traduction de Zerahyah peut être conforme à la leçon attestée dans les manuscrits d'Ankara et d' Istanbul, mais non à celle du manuscrit de Leyde. La même leçon constituait indéniablement la source de la traduction latine. Il en est ainsi en XVIII(XIX), 150,77 pour corporea: ha-garmī / al-jirmiyy ${ }^{121}$. En $\operatorname{XIX}(\mathrm{xx}), 156,1$, la présence du mot essentia dans la traduction latine - mot auquel rien ne correspond dans le manuscrit de Leyde - n' est pas seulement confirmée par māhiyya dans les manuscrits d'Ankara et d' Istanbul, mais aussi par mahūt dans la traduction arabo-hébraïque ${ }^{122}$. D' autre part, il est clair qu'il faut maintenir (avec neuf des dix témoins de l'édition Pattin) dans

120 C'est ce qui ressort du colophon que $M$. Rothschild nous communique d'après le ms. Londres, (olim) Jews' College 42, f. 224: zeh mah še-māṣānū kātūb me-êllū ha-šéārìm benushă’ōt ha-Yišme ēlèm we-ha-noșrīm («Voilà ce que nous avons trouvé de ces chapitres dans les versions des Arabes et des Latins»). Zerahyah aurait ainsi recouru lui-même à la traduction latine, voire à la traduction latino-hébraïque de Hillel de Vérone, sans doute antérieure à la sienne; en tout cas les deux hommes se connaissaient (Rothschild 2013, p. 51). Toutefois, M. Rothschild nous a signalé que la question de savoir si ce colophon est de la main du traducteur ou d'un copiste n' a, à sa connaissance, jamais été approfondie depuis Steinschneider et Bardenhewer, le premier favorisant l'hypothèse du traducteur lui-même, le deuxième celle du copiste (Steinschneider 1863, Hebräische Bibliographie 6, p. 111 [référence empruntée à Bardenhewer 1882, p. 307, n. 1]; Bardenhewer 1882, p. 307, n. 1), à moins que Schreiber en ait discuté dans l'introduction (écrite en hongrois) à son édition de la traduction de Zerahyah (mais ni M. Rothschild ni nous-même ne connaissons la langue hongroise).

121 Voir Rothschild 1994, p. 46o, no .7 . Rothschild remarque à juste titre que cette notion de «corporel» va dans le même sens que l' affirmation de Proclus, qui figure comme source d' inspiration du passage du Liber de causis, voir Proclus, The Elements of Theology, Proposition 111, p. 98, l. 29 (somatikai). Taylor 1981, p. 218, l. 5 [Bāb 18, l. 12], a donc à juste titre préféré la leçon al-jirmiyya, attestée dans les manuscrits d'Ankara et d' Istanbul, non celle de al-hayawānī, présente dans le manuscrit de Leyde.

122 Rothschild 1994, p. 462-463, $\mathrm{n}^{\circ}$. 1. Signalons toutefois que l'essai de reconstruction dont témoigne Bardenhewer (voir Bardenhewer 1882, p. 95, n. 4) ne s' avère pas entièrement 
la traduction latine, XXIII(XXIV), 180,55, les mots esse et, après Et non intelligo per esse nisi, sur base, une fois de plus, du témoignage commun des manuscrits arabes d' Ankara et d' Istanbul, d' une part, et de la traduction de Zerahyah, d' autre part ${ }^{123}$.

Un contre-exemple existe cependant, dans lequel Zerahyah paraît au moins en partie confirmer Leyde contre Ankara / Istanbul: là où en XVIII(XIX), 154,95, le latin, en pleine conformité avec la leçon du seul mot al-șüra des manuscrits d'Ankara et d'Istanbul, porte formam (secundum hancformam), Zerahyah utilise comme Leyde deux expressions: u-be-zeh ha-to'ar (yiheyū ha-madregōt ha-śikhliyōt) u-be-zeh ha-qiyyūm ${ }^{124}$.

Autre configuration encore des rapports entre les témoins: les mots et sui complimenti sont sans doute, en accord avec la vaste majorité des manuscrits latins, à supprimer dans la traduction latine, $\operatorname{XXIV}(\mathrm{XXV}), 184,74-75$, car rien n'y correspond dans les trois témoins arabes ni dans la traduction de Zerahyahah ${ }^{125}$.

correct, car il a rendu le terme essentia par gawhar, ce qui correspond davantage à une lecture du latin substantia.

123 Voir respectivement, Taylor 1981, p. 500, n. 179; Rothschild 1994, p. 471, nº. 6).

124 Taylor 1981, p. 220, l. 4-5 [Bāb 18, l. 23], a opté pour la leçon wa-'alā hāḍa l-șifa (...) wa-bihād tion latine et la leçon des manuscrits d'Ankara et d'Istanbul. Toutefois, son choix trouve un support (du moins, partiel) supplémentaire dans la traduction hébraïque de Zerahyah, au moins pour l' addition à la fin des mots wa-bi-hādāa al-qiyās. En effet, l' expression u-be-zeh ha-qiyyūm confirme - moyennant l'hypothèse de Rothschild [Rothschild 1994, p. 461-462, $\mathrm{n}^{\circ} 13$ ] d'une possible lecture fautive du $s$ final de qiyās comme un $m$ - la présence de ces mots dans le modèle du traducteur. Quant à l'autre expression, $u$-be-zeh ha-to'ar, elle peut résulter aussi bien d'une leçon wa-'alā hād $\bar{d} l$-șifa que wa-'ala hā l-șūra, car le terme to’ar couvre en philosophie médiévale un spectre sémantique très large («attribut»; «description, propriété»; «forme»; Klatzkin 1933, t. IV, p. 172-176). L'affirmation de Rothschild (ibid.) selon laquelle la traduction to'ar est «conforme au latin» (forma), est peut-être discutable dans la mesure où le sens primaire de ce mot, à savoir «attribut», correspond davantage à șifa qu' à șüra. Certes, rien n' exclut que to’ar constitue la traduction de șūra, mais il me semble qu'il soit impossible dans ce contexte de fixer avec certitude le terme arabe sous-jacent. Dans ce cas la traduction de Zerahyah ne permet donc pas de fixer avec certitude le texte arabe présent dans son modèle. Reste néanmoins la difficulté de déterminer le rapport précis avec le texte grec de Proclus (Prop. 111, p. 98, l. 31-32). Tout laisse croire que l' auteur du texte arabe du Liber de causis a lu outoos au lieu de outos. L'option de Taylor ne peut pas simplement être écartée, mais nécessite sans doute un examen approfondi entre le texte arabe et le texte grec de Proclus, qui en constitue la source d'inspiration.

125 Voir Rothschild 1994, p. 472, nº. 3. Taylor (Taylor 1981, p. 247, l. 1 [Bāb 24, l. 11]) de façon un peu surprenante, préfère par contre d' ajouter dans le texte arabe, sur la base de la traduction latine, telle que Pattin l'a éditée (mais sans tenir compte du fait que cette lecture est basée sur une minorité infime de ses témoins), wa-tamāmuhu. 
Enfin, nous souhaitons attirer tout particulièrement l' attention sur deux cas:

En v(VI), 58,28-30, la traduction latine rend «quod est quoniam causa prima non cessat illuminare causatum suum et ipsa non illuminatur a lumine alio», alors que les trois témoins arabes portent: wa-d̄àlika anna al-illa allatì tunìru awwalan tunìru ma'lūlahā wa-hiya lā tastanìru min nūrin āharin («En effet, la cause, qui illumine de façon primordiale, illumine ce qu' elle a causé, alors qu' elle n' est pas illuminée par une autre lumière $)^{126}$. La traduction de Zerahyah, qui porte: we-zeh kì ha-illāh ha-rišōnāh 'ôśáăh ba-'ālūlāh we-hēm èynām 'ósím bāh («c' est que la cause première agit dans son causé et eux (sic) n' agissent pas en elle »127) a en commun avec le latin l' expression «première cause ». Cette concordance peut difficilement être le fruit du hasard. Elle pourrait indiquer l' existence d' un modèle commun aux deux traductions, qui offrait une autre lecture de l'arabe. L'épitomé de 'Abd al-Lāṭif al-Baġdādī est un témoin incontestable pour la confirmation de cette correspondance, car on y lit: li-anna al-'illa al-ülà tunìru kulla 'illatin wa-ma'lūlin wa-hiya là tastanìru min nūrin äharin («Car la cause première illumine chaque cause et chaque causé, alors qu' elle n' est pas illuminée par une autre lumière $\left.{ }^{128}\right)$. On y trouve clairement exprimé que c'est la cause première qui illumine, mais on y cherche en vain l'équivalent de l'idée selon laquelle cette illumination est sans interruption - idée, par ailleurs, aussi absente de la traduction de Zerahyahah ${ }^{129}$. Étant donné qu' al-Bag̉dādī mentionne une illumination de l'ensemble des causes et des choses causés (en reformulant ainsi, et en toute probabilité, d'une façon personnelle l'expression ma'lūlahā, «ce qu' elle a causé»), il n' est la source directe ni de la traduction latine ni de celle de Zerahyah. Cependant, cela ne rend pas impossible l' existence d'une source commune arabe, qui aurait été connue du traducteur latin et de Zerahyah (même si ce dernier exprime en termes d' «agir» ce que le latin, en conformité avec tous les témoins arabes, présente en termes d' «illuminer»). En effet, il suffit de supposer que cette source recelait une formulation très similaire à celle d' al-Bag̉dādī, tout en conservant, avec tous les témoins majeurs, la leçon ma'lülahā. On ne peut certes pas exclure que Zerahyah se soit appuyé directement sur le latin. La question qui se poserait alors serait celle de savoir pourquoi il a délaissé l' idée de 'permanence' qui qualifie, dans la version latine, l' activité (illuminatrice) divine.

\footnotetext{
126 Taylor 1981, p. 16o, l. 6-161, l. 1 [Bāb 5, l. 6-7].

127 Rothschild 1994, p. 427, $\mathrm{n}^{\circ} .3$.

128 Badawi 1955, p. 249, l. 4.

129 Taylor (Taylor 1981, p. 451, n. 41) suggère l'existence de l'affirmation suivante dans le modèle du traducteur latin: (wa-dāalika anna) al-illa al-ūlà lā tuzālu tunìru málūlahā.
} 
En VIII (IX), 84,75, le latin et horizontem naturae est confirmé par la traduction de Zerahyah, qui a be-ōfan (ou:be-ofeq, de même sens) ha-țeba' (l'horizon de la nature $)^{130}$, mais ne correspond nullement à la lecture des témoins arabes, qui portent invariablement wa-mā fawqu al-țabīa («au-dessus de la nature ${ }^{131}$ ). Cette dernière leçon semble être confirmée par un des témoins indirects arabes, à savoir le Liber de causis II, où manque toutefois, immédiatement après, la formule assez redondante: fa-innahä fawqu al-țabīa ( «car elle est audessus de la nature» $)^{132}$. Comme Taylor et Rothschild le supposent, la variante wa-ufuq al-țabǐa figurait sans doute dans le modèle utilisé tant par Gérard de Crémone que par Zerahyahah ${ }^{133}$. À l' appui d'une telle lecture on pourrait évoquer l' affirmation en II, 22, 81-82 que l'âme est in horizonte aeternitatis inferius, mais seul un examen approfondi du passage pourrait révéler la plausibilité de cette hypothèse $\mathrm{e}^{134}$. Quoi qu'il en soit, la traduction hébraïque de Zerahyah montre qu' on n' a certainement pas le droit d'intervenir dans le texte de la traduction latine, et cela d'autant plus qu' on ne peut pas totalement exclure que Zerahyah ait eu recours - fût-ce de façon partielle - à la traduction latine. Le véritable enjeu est de savoir si sa leçon «horizon de la nature» est basée sur une lecture fautive ou, tout au contraire, correcte de l'arabe.

\section{$9 \quad$ Conclusion}

Malgré les œuvres pionnières, et extrêmement méritoires, de Bardenhewer, Pattin, Vansteenkiste et, en dernier lieu, Taylor, il reste un long travail à accomplir avant que l' on puisse songer à élaborer une véritable édition critique de la traduction latine, qui par ailleurs restera incontestablement très dépendante d'une véritable édition critique du texte arabe. Dans ce qui précède, nous avons essayé de dresser un petit tableau de quelques défis majeurs et de clarifier quelques problèmes particuliers. Ce survol est loin d'être exhaustif. En effet, nous nous sommes souvent limités à l' examen de quelques cas exemplaires. Mais nous n' avons pas prêté attention aux deux cas de translittération, à savoir des mots arabes 'aql et hilya, chacun transcrit sous une multitude de formes,

\footnotetext{
130 Rothschild 1994, p. 439, nº. 8.

131 Taylor 1981, p. 177, l. 5 [Bāb 8, l. 19].

132 Liber de causis II, p. 346, 1. 4-5.

133 Respectivement Taylor 1981, p. 46o, n. 70; Rothschild 1994, p. 439, nº 8.

134 La notion d' «horizon» dans ce contexte n' est pas inspirée de Proclus, mais plutôt dérivée de Plotin; cf. D'Ancona 2014, p. 144, n. 44.
} 
attestées parfois dans le même manuscrit ${ }^{135}$. Nous n' avons pas discuté non plus des quelques cas où seule une analyse approfondie pourrait éclairer ce qui à première vue constitue une non-conformité entre le texte arabe et la traduction latine ${ }^{136}$. Nous espérons néanmoins avoir démontré la complexité de la tâche qu' il reste à accomplir: d'une part, fixer aussi bien que possible la traduction latine originale telle qu' elle figurait dans l' archétype; d'autre part, identifier la formulation de cette traduction telle qu' elle était accessible, à un moment précis et dans un lieu donné, à ses lecteurs.

\section{Bibliographie}

\section{Manuscrits consultés}

Admont, Stiftsbibliothek, cod. 405

Angers, BM $45^{\circ}$

Ankara, İsmail Saib I 1696

Aoste, Seminario maggiore $71\left(\mathrm{olim} \mathrm{Ai}^{\circ} \mathrm{D} 2 \mathrm{O}\right)$

Assise, BC

298

663

Berlin, Staatsbibliothek, M 1494, Lat. Qu. 449

Bologne, Bibl. Univ. 2344

Bordeaux, B M 421

Bruges,

Bibliothèque de la Ville

463

478

BC 102/125

135 L'analyse de ses différentes formes pourra sans doute contribuer à l'établissement du stemma codicum.

136 Il va de soi qu'il y faut ajouter l'étude du lexique utilisé. L'importance de celle-ci s'illustre très bien à partir de l'utilisation de l'adverbe simul en XXII (XXIII), 175, 14: simul omnes

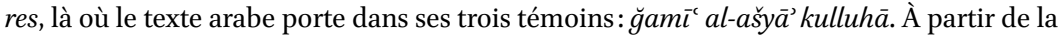
traduction latine, Taylor (Taylor 1981, p. 238, l. 2 [Bāb 22, l. 17]) a corrigé le texte arabe en ğamī al-ašy $\bar{a}^{\prime} m a^{\prime} a n$. Mais une telle correction ne s'impose nullement si on tient compte du fait que simul traduit à plusieurs reprises ğamī'an dans le De anima de l' Avicenne latin (Avicenna latinus 1972, p. 303, $\mathrm{n}^{\circ} .123$ et 1968, p. 223, $\mathrm{n}^{\circ}$. 108). Il est vrai que dans ce dernier, l' arabe porte l' adverbe, mais le traducteur latin du Liber de causis peut avoir opté pour l'utilisation de la forme adverbiale pour rendre le substantif $\breve{g} a m \bar{\iota}^{c}$, dans un contexte où apparait immédiatement après la notion de tout(es). 
Bruxelles, Bibliothèque royale II, 2314

Cava de' Tirreni (Salerne), B. ss. Trinità 31

Césène, Bibl. Malatestiana, Ms. Plut. XxII, Dext. 6

Chicago, Vault Case 23

Cologny, Bodmer Св 10

Erfurt, $\mathrm{Ca}$

2-00363

4-00018

Évreux, вм 79

Firenze, Bibl. Laur., Ashburnham 1674

Genève, BGE, Lat. 76

Istanbul, Sülemanyie Kütüphanesi, Hacı Mahmud 5683

Leipzig, Universitätsbibliothek

1339

1382

Leyde, Or. 209

Londres, olim Jews' College, olim Beth Din and Beth Hamidrash 42

Madrid, BN 489

München, вSв $\mathrm{Clm} 527$

Oxford, Bodl., Selden sup. 24

Paris, BnF,

lat. 6318

lat. 6569

lat. 16082

Rennes, BM 149

Rouen, BM 920

Sankt Gallen, Stiftsbibliothek, Cod. Sang. 837

Toledo, Bibl. del Cabildo 97-1

Tours, BM 68 o

Troyes, 1374

Vaticano (Città del),

Ottob. lat. 1415

Urb. lat. 206

Vat. lat.

2089

14717

20984

Wien, ÖNB 2491 


\section{Sources primaires}

al-Amirī - voir Wakelnig 2006

al-Bag̉dādī - voir Badawi 1955b

Albert le Grand, De causis et processu universitatis a prima causa, éd. W. Fauser, Münster, Aschendorff, 1993.

Avicenna latinus, Liber de anima seu sextus de Naturalibus, IV-v, éd. S. Van Riet, Louvain / Leiden, Éditions orientalistes / Brill, 1968.

Avicenna latinus, Liber de anima seu sextus de Naturalibus, I-III, éd. S. Van Riet, Louvain / Leiden, Peeters / Brill, 1972.

Avicenna latinus, Liber de philosophia prima sive scientia divina, I-IV, éd. S. Van Riet, Louvain / Leiden, Peeters / Brill, 1977.

Avicenna latinus, Liber de philosophia prima sive scientia divina, V-X, éd. S. Van Riet, Louvain / Leiden, Peeters / Brill, 198o.

Avicenna latinus, Liber de philosophia prima sive scientia divina, I-X. Lexiques, éd. S. Van Riet, Louvain-la-Neuve / Leiden, Peeters / Brill, 1983.

Avicenna latinus. Liber primus naturalium. Tractatus primus. De causis et principiis naturalium, éd. S. Van Riet, Louvain / Leiden, Éditions orientalistes / Brill, 1992.

Bacon, R., Quaestiones super Librum de causis, éd. R. Steele, Oxford, Clarendon Press, 1935 .

Gilles de Rome, Super librum De causis, Venetiis 1550, reprint Frankfurt, Minerva, 1968. Ibn Sab’̄̄n, Budd al-'ārif, éd. Ğūrğ Katturah, Beyrouth, Dār al-Andalus, Dār al-Kindī, 1978.

Ibn Sab'īn, Kalām 'alā l-masāil al-șiqiliyya, éd. Ş. Yaltkaya. Beyrouth, Al-Mațba’a alkāthūlīkiyya, 1941. Traduction allemande A.A. Akasoy, Philosophie und Mystik in der späten Almohadenzeit. Die sizilianischen Fragen des Ibn Sabīn, Leiden / Boston, Brill, 20o6. Traduction italienne P. Spallino, Ibn Sabīn. Le Questione siciliane. Frederico II e l'universo filosofico, Palermo, Officina di Studi Medievali, 2002.

Liber de causis

Éditions du texte arabe: Badawi - voir Badawi 1955a; O. Bardenhewer, Die pseudoaristotelische Schrift Ueber das reine Gute bekannt unter dem Namen Liber de causis, Freiburg im Breisgau, Herder'sche Verlag, 1882, p. 58-118; R.C. Taylor, The Liber de causis: (Kalām fī maḥ̣ al-khair): A Study of Medieval Neoplatonism, Toronto, University of Toronto (thèse de doctorat dactilographiée), 1981, p. 136-271.

Éditions de la traduction latine médiévale: Bardenhewer, Die pseudo-aristotelische Schrift, p. 120-302, p. 163-191; A. Pattin, «Le Liber de causis. Édition établie à l' aide de 90 manuscrits», dans Tijdschrift voor Philosophie, 28(1966), p. 90-203; repr. dans A. Pattin, Miscellanea. I. Liber de causis, Leuven, Bibliotheek van de Faculteit der Godgeleerdheid, 2ooo. Traduction française P. Magnard, O. Boulnois, B. Pinchard, J.L. Solère, La demeure de l'être. Autour d'un anonyme. Étude et traduction du Liber de causis, Paris, Vrin, 1994. Traduction allemande A. Fidora, A. Niederberger, Von Bag- 
dad nach Toledo: das Buch der Ursachen und seine Rezeption im Mittelalter, Mainz, Dieterich, 2001.

Édition de la traduction hébraïque de Zerahyah : Pseudo-Aristoteles Liber de causis Zerachja b. Izsák, éd. I. Schreiber, Budapest, Athenaeum, 1916.

Liber de causis II - voir P. Thillet, S. Oudaimah, «Proclus arabe. Un nouveau Liber de causis?», dans Bulletin d'Études Orientales 53-54 (2001-2002), p. 293-368.

Proclus, Tria Opuscula (De providentia, libertate, malo). Latine Guilelmo de Moerbeke vertente et Graece ex Isaach Sebastocratoris aliorumque scriptis collecta, éd. H. Boese, Berlin, de Gruyter, 196o.

Proclus, The Elements of Theology. A Revised Text with Translation, Introduction and Commentary by E.R. Dodds, Second edition, Oxford, Clarendon Press, 1963.

Thomas d'Aquin, Super Librum de causis expositio, éd. H.-D. Saffrey, Fribourg, Société philosophique, 1954.

\section{Sources secondaires}

Akasoy, A.A. (2006) - voir Ibn Sab'īn.

Badawi, A. (1955), Al-Aflațūnōya al-muhdața 'inda al-'Arab. Neoplatonici apud Arabes, Le Caire, Maktaba al-Naḥ̣̣a al-Mișrīya.

Badawi, A. (1955a), «Kitāb 'al-īdāḥ fī ḩayr al-maḥḍ li-Arisṭuṭālīs», dans Badawi 1955, p. 1-33.

Badawi, A. (1955b), «Min kitāb 'Fì mā ba'd al-țabīa' li-'Abd al-Lațîf Yusūf al-Bag̉dādī», dans Badawi 1995, p. 248-256.

Badawi, A. (1974), «Risālat al-Aflațūn al-ilāhī fì al-radd 'alā man qāla an al-insān talāšì wa-fanī», dans Id., Al-Aflațūn fì al-Islām (Platon en pays d'Islam), Téhéran, Institute of Islamic Studies McGill University, Tehran Branch, p. 337-341.

Bardenhewer, O. (1882) - voir Liber de causis

Calma, D. (éd.) (2016), Neoplatonism in the Middle Ages. Vol. I. New Commentaries on Liber de causis (ca. 1250-1350). Vol. II. New Commentaries on Liber de causis and Elementatio theologica (ca. 1350-1500), Turnhout, Brepols.

Calma, D. (2016a), «The Exegetical Tradition of Medieval Neoplatonism. Considerations on a Recently Discovered Corpus of Texts », in Calma 2016-I, p. 11-52.

Carron, D. (2016b), «A Theological Reading of the Liber de causis on the Turn of the Fourteenth century: The Example of William of Leus », in Calma 2016-I, p. 467-549.

Cruz Hernández, M. (1951), «El Fontes Quaestionum ('Uyūn al-masā̉il) de Abū Nașr alFārābī », dans Archives d'Histoire Doctrinale et Littéraire du Moyen Âge 18, p. 303-323.

D’Ancona, C. (1995), «La doctrine néoplatonicienne de l'être entre l'Antiquité tardive et le Moyen Âge. Le Liber de causis par rapport à ses sources», in Id., Recherches sur le Liber de causis, Paris, Vrin, p. 121-153.

D'Ancona, C. (2014), «The Liber de causis», in S. Gersh (éd.), Interpreting Proclus. From Antiquity to the Renaissance, Cambridge, Cambridge University Press, p. 137-161. 
de Biberstein Kazimirski, A. (186o), Dictionnaire arabe-français. Paris, Maisonneuve, 1860.

Gaffiot, F., nouv. éd. P. Flobert (200o). Dictionnaire latin-français. Paris, Hachette.

Fidora, A., Harvey Hames, J., Schwartz, Y. (éds) (2013), Latin into Hebrew: Texts and Studies. Vol. II. Texts in Contexts, Leiden / Boston, Brill.

Fidora, A., Niederberger A. (2001) - voir Liber de causis.

Fuchs, J.W., Weijers, O., Gumbert, M. (éds) (1977-2005), Lexicon Latinitatis Nederlandicae Medii Aevi, Leiden, Brill.

Janssens, J. (2002), «L'Avicenne latin: particularités d'une traduction», dans J. Janssens, D. De Smet (éds), Avicenna and His Heritage, Leuven, Leuven University Press, p. 113-129.

Klatzkin, J. (1928-1933), Thesaurus philosophicus linguae hebraicae et veteris et recentioris, Berlin, Eshkol.

Latham, E., Howlett, R.D., Ashdowne, R.K. (éds) (1975 sqq.), Dictionary of Medieval Latin from British Sources. Oxford, British Academy.

Martini-Bonadeo, C. (2013), 'Abd al-Lațīf al-Baġdādì’s Philosophical Journey. From Aristotle's Metaphysics to the 'Metaphysical Science', Leiden / Boston, Brill.

Lator, E. (1944), «Ibn Sab'īn de Murcia y su Budd al-'ārif. Fuentes y bibliografía », dans Al-Andalus 9, p. 371-417.

Magnard, P., Boulnois, O., Pinchard, B., Solère, J.-L. (1994) - voir Liber de causis.

Meliadò, M. (2016), «Le Questiones super Librum de Causis attribué à Johannes Wenck. Concezione, fonti e tradizione manoscritta del commento », in Calma 2016-II, p. 225270.

Niermeyer, J.F., comp. van De Kieft, C. adiuvante G.S.M.M. Lake-Schoonebeek (ed) (1993), Mediae Latinitatis Lexicon Minus. Abbreviationes et Index Fontium. Leiden / New York / Köln, Brill.

Pattin, A. (1966), voir Liber de causis- Éditions de la traduction latine.

Prinz, O., Antony, H., Wellhausen, A. (éds) (1959sqq.), Mittellateinisches Wörterbuch bis zum ausgehenden 13. Jahrhundert. München, Beck.

Rothschild, J.-P. (1994), «Les traductions du Livre des Causes et leur copies », dans Revue d'histoire des textes 24, p. 393-484.

Rothschild, J.-P. (2013a), «Le Livre des causes du latin à l'hébreu: textes, problèmes, réception », dans Fidora, Harvey, Schwartz, p. 47-84.

Rothschild, J.-P. (2013b), «Les traductions hébraïques du Livre des causes latin, édition synoptique», dans Fidora, Harvey, Schwartz, p. 289-367.

Rothschild, J.-P. (2018), Moïse b. Sabbataï, lecteur juif du Livre des causes et adversaire de la kabbale, en Italie, vers 1340, Turnhout, Brepols.

Schreiber, I. (1916) - voir Liber de causis.

Spallino, P. (2002) - voir Ibn Sab’īn.

Taylor, R.C. (1981) - voir Liber de causis. 
Taylor, R.C. (1982), «Neoplatonic Texts in Turkey: Two Manuscripts Containing Ibn Ṭufayl's Hayy ibn Yaqzān, Ibn al-Sīd's Kitāb al-Hadā’iq, Ibn Bājja's Ittiṣāl al-'Aql bil-Insān, the Liber de causis and an Anonymous Neoplatonic Treatise on Motion», dans Mélanges de l'Institut Dominicain d'Études Orientales 15, p. 251-264.

Taylor, R.C. (1983), «The Liber de causis: A Preliminary List of extant Mss», dans Bulletin de philosophie médiévale 25, p. 63-84.

Taylor, R.C. (1989), « Remarks on the Latin Text and the Translator of the Kaläm fimahd al-khair/ Liber de causis», dans Bulletin de philosophie médiévale 31, p. 75-102.

Taylor, R.C. (2016), «Primary and Secondary Causality», dans R.C. Taylor, L.X. LópezFarjeat (éds), The Routledge Companion to Islamic Philosophy, London / New York, Routledge, p. 225-235.

Thesaurus linguae latinae (19oosqq.), München / Berlin, Saur / de Gruyter.

Thillet, P., Oudaimah, S. (2001-2002) - voir Liber de causis II.

Türker, M. (1965), «Fârâbî̀ye atfedilen küçük bi eser», dans Araştirma (Ankara) 3, p. 163.

Van Riet, S. (1963), «La traduction latine du De anima d'Avicenne. Préliminaires à une édition critique», dans Revue philosophique de Louvain 61, p. 583-626.

Vansteenkiste, C. (1967), «Intorno al testo latino del Liber de causis», dans Angelicum 44, p. $6 o-83$.

Wakelnig, E. (2006), Feder, Tafel, Mensch. Al-Āmirīs Kitāb al-Fuṣul fĩ l-Ma'ālim al-ilāhīya und die arabische Proklos-Rezeption in 10.Jh., Leiden / Boston, Brill. 